TI 2015-040/III

Tinbergen Institute Discussion Paper

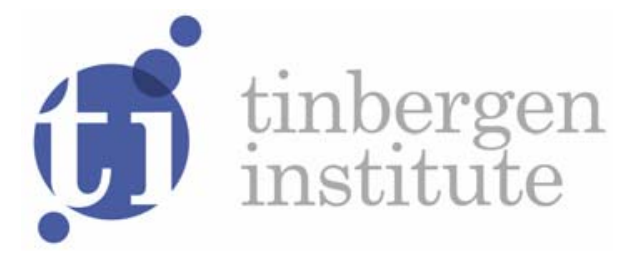

\title{
Testing for Stock Market Contagion: A Quantile Regression Approach
}

Sungyong Park'

Wendun Wang ${ }^{2}$

Naijing Huang ${ }^{3}$

' Chung-Ang University, Seoul, Korea;

2 Erasmus School of Economics, Erasmus University Rotterdam, and Tinbergen Institute, the Netherlands;

${ }^{3}$ Boston College, United States. 
Tinbergen Institute is the graduate school and research institute in economics of Erasmus University Rotterdam, the University of Amsterdam and VU University Amsterdam.

More TI discussion papers can be downloaded at http://www.tinbergen.nl

Tinbergen Institute has two locations:

Tinbergen Institute Amsterdam

Gustav Mahlerplein 117

1082 MS Amsterdam

The Netherlands

Tel.: +31(0)205251600

Tinbergen Institute Rotterdam

Burg. Oudlaan 50

3062 PA Rotterdam

The Netherlands

Tel.: +31(0)10 4088900

Fax: $+31(0) 104089031$

Duisenberg school of finance is a collaboration of the Dutch financial sector and universities, with the ambition to support innovative research and offer top quality academic education in core areas of finance.

DSF research papers can be downloaded at: http://www.dsf.nl/

Duisenberg school of finance

Gustav Mahlerplein 117

1082 MS Amsterdam

The Netherlands

Tel.: +31(0)20 5258579 


\title{
Testing for Stock Market Contagion: A Quantile Regression Approach*
}

\author{
Sung Y. Park ${ }^{\dagger}$ \\ Wendun Wangt \\ Naijing Huang ${ }^{\S}$
}

This version: March 25, 2015

\begin{abstract}
Regarding the asymmetric and leptokurtic behavior of financial data, we propose a new contagion test in the quantile regression framework that is robust to model misspecification. Unlike conventional correlation-based tests, the proposed quantile contagion test allows us to investigate the stock market contagion at various quantiles, not only at the mean. We show that the quantile contagion test can detect a contagion effect that is possibly ignored by correlation-based tests. A wide range of simulation studies show that the proposed test is superior to the correlation-based tests in terms of size and power. We compare our test with correlation-based tests using three real data sets: the 1994 Tequila crisis, the 1997 Asia crisis, and the 2001 Argentina crisis. Empirical results show substantial differences between two types of tests.
\end{abstract}

Keywords: Financial contagion, Quantile regression, One-sided score test

*We are grateful to the participants at the WISE lunch seminar, the Midwest Econometrics Group (MEG) 2009, the seminar at Southwestern University of Finance and Economics for their useful comments. We are responsible for any remaining errors.

${ }^{\dagger}$ School of Economics, Chung-Ang University, 84 Heukseok-Ro, Dongjak-Gu, Seoul, Korea. E-mail: sungparkecau.ac.kr

${ }^{\ddagger}$ Econometric Institute, Erasmus University Rotterdam and Tinbergen Institute. E-mail: wang@ese. eur.nl

${ }^{\S}$ Department of Economics, Boston College. E-mail: huangnaijing@hotmail . com 


\section{Introduction}

Financial crises occurred throughout the world in the second half of the 1990s; these include the 1994 Tequila crisis, the 1997 Asian flu, and the 2001 Argentina crisis. These crises have stimulated many empirical studies on contagion between stock markets, among which testing the occurrence of contagion has been of particular interest in both theoretical and empirical senses.

Various tests for financial contagion have been proposed according to various definitions of contagion; see Forbes and Rigobon (2001)(pp. 44-47) for a comparative review of these definitions. One popular definition of contagion is a significant increase in crossmarket linkages after a shock to the original country (Forbes and Rigobon, 2001). Typically, this linkage is measured by the (mean) correlation of the asset returns; early influential studies using correlation include King and Wadhwani (1990) and Lee and Kim. Forbes and Rigobon (2002) showed that the heteroscedastic behavior of asset returns causes a bias in the correlation-based test and suggested an adjusted correlation measure to avoid such bias. Another definition is based on conditional probability: financial contagion is described as the joint occurrence of the extreme values (Bae et al., 2003). Other tests for financial contagion include the vector autoregression (VAR) approach (Faveroa and Giavazzi (2002), Pesaran and Pick (2007)), the copula method with Markov switching parameters (Rodriguez, 2007), and the quantile-based measure of co-movement (Cappiello et al., forthcoming). Dungey et al. (2005a) compared various tests and related them in a pooled regression framework.

In this paper, we propose to test contagion based on "quantile correlation". In contrast to the mean correlation, the quantile correlation captures the linkage of stock returns 
not only at the average (mean) level but also at different quantiles. The linkage at the lower quantiles is of particular interest for risk-averse agents since they are more concerned with whether the slump of one stock market would have an impact on another. The lower quantile linkage is also crucial for policy makers because it may influence government decisions and actions in the crisis period. In addition, we show that tests based on mean correlation may not detect the change of the linkage in some cases, and thus they may suffer from a poor power property. Our quantile correlation test resembles the probability-based test in that both methods attempt to model the behavior of stock returns at lower quantiles. However, the probability-based tests only focus on the exceedences, a small proportion of the support to the whole distribution, while regression quantiles are obtained using the whole sample.

This paper makes three main contributions. First, we contribute to the econometric literature by proposing two-sided and one-sided score tests for the slope coefficient of the quantile regression in a likelihood framework and study their asymptotic properties. The proposed score tests are robust under possible distributional misspecification and are easy to implement since they only require estimators under the null hypothesis, especially for the one-sided test. Second, we contribute to the empirical finance literature by providing new insight into testing financial contagion. The proposed quantile contagion test allows us to investigate the various impacts of one country's asset returns on those of others at various quantile levels. As we have mentioned, standard correlation-based tests can only indicate the linkage between two asset returns on an average level. However, the risk-averse agents and policy makers are typically more concerned with the linkage at the lower quantiles, and it can also be useful to consider the linkage at the upper quantiles. By implementing our quantile contagion test, one could study whether contagion exists 
when a stock market is experiencing a slump or advance. We compare the size and power property of our proposed tests with three forms of Forbes and Rigobon's correlationbased tests $(F R)$, the most popular contagion tests, in various simulation designs. We find that the quantile contagion test beats the $F R$ tests in most cases, especially when the data are skewed and leptokurtic. Finally, we conduct an empirical analysis of the contagion effect in the Tequila crisis, the Asian crisis, and the Argentine crisis and compare our tests with those of the correlation-based tests. The quantile contagion test generally produces different results and new insights. For example, we find that in most transmission channels of the Tequila crisis and the Argentine crisis FR tests show no financial contagion at mean values, while the quantile contagion test reports significant contagion effects at lower quantiles. In contrast, $F R$ tests report contagion in some transmission channels in the Asian crisis, while the quantile contagion test indicates that contagion may only happen on an average level rather than at all quantiles. We conclude that no contagion in the mean correlation does not imply no contagion in the other quantiles. Therefore, when investors and policy makers study the joint behavior of multiple stock returns they should consider the different quantiles in addition to the average level.

This paper is organized as follows. The next section presents our model setup and compares it to other models in the literature. In Section 3, we derive the symmetric test as a preliminary of the contagion test. The contagion test procedure is performed in Section 4. We conduct Monte Carlo experiments in Section 5, and provide the empirical analysis in Section 6. Finally, Section 7 concludes. 


\section{Model Setup}

To simplify the model without loss of generality, we only consider the relationship between two asset markets. Following Dungey et al. (2005a) we denote the asset returns of country 1 over the tranquil period as $x_{1, t}$ and the asset returns of country 2 over the tranquil period as $x_{2, t}$. We also denote the two countries' asset returns in the turbulent period as $y_{1, t}$ and $y_{2, t}$. Then the relationship between the two asset returns in each regime follows

$$
\begin{aligned}
& x_{2, t}=\alpha_{0}+\alpha_{1} x_{1, t}+\eta_{x, t}, \\
& y_{2, t}=\beta_{0}+\beta_{1} y_{1, t}+\eta_{y, t},
\end{aligned}
$$

where $\alpha_{1}$ and $\beta_{1}$ capture the degree of the influence of country 1's asset returns on those of country 2 over the tranquil and volatile periods, respectively, and $\eta_{x, t}$ and $\eta_{y, t}$ are corresponding i.i.d. innovations. This is the standard static setup in the literature (see, for example, Dungey et al., 2005), and it assumes that the observations are serial uncorrelated. Later, we shall relax this assumption and incorporate the dynamic feature, at least to some extent, by including lagged variables.

From (2.1) and (2.2), it can be easily checked that contagion is not detected if $\alpha_{1}=\beta_{1}$, that is, if country 1's returns have the same effect on country 2's during non-crisis and crisis periods. In contrast, if $\alpha_{1}<\beta_{1}$, then country 2 is tied to country 1 to a larger extent in the volatile periods, and thus we find the occurrence of contagion. Therefore, the null and alternative hypotheses for testing for contagion are, respectively,

$$
H_{0}: \alpha_{1}=\beta_{1} \quad \text { and } \quad H_{A}: \alpha_{1}<\beta_{1}
$$

The test can be performed based on the sample (mean) correlation of the two asset re- 
turns in two regimes, which are denoted by $\hat{\rho}_{x}$ and $\hat{\rho}_{y}$, respectively (King and Wadhwani, 1990; Forbes and Rigobon, 2002)

$$
\hat{\rho}_{x}=\frac{\hat{\sigma}_{x, 12}}{\sqrt{\hat{\sigma}_{x, 1}^{2} \hat{\sigma}_{x, 2}^{2}}}=\hat{\alpha}_{1} \hat{\sigma}_{x, 1}, \quad \hat{\sigma}_{x, 2}=\frac{\hat{\sigma}_{y, 12}}{\sqrt{\hat{\sigma}_{y, 1}^{2} \hat{\sigma}_{y, 2}^{2}}}=\hat{\beta}_{1} \frac{\hat{\sigma}_{y, 1}}{\hat{\sigma}_{y, 2}},
$$

where $\hat{\sigma}_{x, i}$ and $\hat{\sigma}_{y, i}$ denote the standard error of country $i^{\prime}$ s asset returns in the non-crisis and crisis periods, respectively. Interestingly, Forbes and Rigobon (2002) showed that the sample correlation is biased in the sense that $\rho_{y}>\rho_{x}$ even when $\alpha_{1}=\beta_{1}$ because of $\sigma_{y, 1}>\sigma_{x, 1}$. To avoid a spurious test, they proposed an adjusted estimator of $\rho_{y}$

$$
\hat{v}_{y}=\frac{\hat{\rho}_{y}}{\sqrt{1+\left(\frac{\hat{\sigma}_{y, 1}^{2}-\hat{\sigma}_{x, 1}^{2}}{\hat{\sigma}_{x, 1}^{2}}\right)\left(1-\hat{\rho}_{y}^{2}\right)}} .
$$

Thus, the null hypothesis of the test is $H_{0}: \hat{\rho}_{x}=\hat{v}_{y}$, and it can be tested using the $t$ statistic

$$
F R=\frac{\hat{v}_{y}-\hat{\rho}_{x}}{\sqrt{\frac{1}{T_{x}}+\frac{1}{T_{y}}}}
$$

where $T_{x}$ and $T_{y}$ are the numbers of observations in the tranquil and turmoil regimes, respectively. The asymptotic variance of the estimator $\hat{v}_{y}-\hat{\rho}_{x}$ is approximated by $T_{x}+$ $T_{y}$ under the assumption of independence. Forbes and Rigobon (2002) proposed three alternative forms of the $F R$ test to improve the asymptotic approximation using Fisher's Z-transformation

$$
F R_{1}=\frac{\frac{1}{2} \ln r_{y}-\frac{1}{2} \ln r_{z}}{\sqrt{\frac{1}{T_{2}-3}+\frac{1}{T-3}}}, \quad F R_{2}=\frac{\frac{1}{2} \ln r_{y}-\frac{1}{2} \ln r_{x}}{\sqrt{\frac{1}{T_{2}-3}+\frac{1}{T_{1}-3}}}, \quad F R_{3}=\frac{\frac{1}{2} \ln r_{y}-\frac{1}{2} \ln r_{z}}{\sqrt{\frac{1}{T_{1}-3}-\frac{1}{T-3}}} .
$$

where $r_{y}:=\left(1+\hat{v}_{y}\right) /\left(1-\hat{v}_{y}\right), r_{z}:=\left(1+\hat{\rho}_{z}\right)\left(1-\hat{\rho}_{z}\right)$, and $r_{x}:=\left(1+\hat{\rho}_{x}\right)\left(1-\hat{\rho}_{x}\right)$.

Note that $\alpha_{1}$ and $\beta_{1}$ coincide with the adjusted correlations $\rho_{x}$ and $v_{y}$ if we scale the asset returns of the individual countries in (2.1) and (2.2) by their corresponding standard 
deviations of the tranquil period $\sigma_{x, 1}$ and $\sigma_{x, 2}$, respectively. Thus, we can test contagion using the regression coefficients $\alpha_{1}$ and $\beta_{1}$.

Next, we consider the following pooled regression model proposed by Dungey et al. (2005a)

$$
z_{2, t}=\gamma_{0}+\gamma_{1} d_{t}+\gamma_{2} z_{1, t}+\gamma_{3} z_{1, t} d_{t}+\eta_{t}
$$

where $z_{i, t}=\left(x_{i, 1}, x_{i, 2}, \ldots, x_{i, T_{x}}, y_{i, 1}, y_{i, 2}, \ldots, y_{i, T_{y}}\right)^{\prime}, i=1,2$, and $d_{t}$ is the dummy variable such that $d_{t}=1$ for the volatile period and $d_{t}=0$ otherwise. Note that $\gamma_{3}$ is identical to $\beta_{1}-\alpha_{1}$ in (2.1) and (2.2). Thus, the contagion test is simplified to test whether $\gamma_{3}$ is larger than zero. Moreover, (2.3) can generalize the contagion test by allowing multivariate countries. The hypotheses for testing contagion in the pooled equation are

$$
H_{0}: \gamma_{3}=0 \text { and } H_{A}: \gamma_{3}>0
$$

Considering the possibility of serial dependent innovations, the above models (2.1), (2.2), and (2.3) can be readily extended by adding lagged variables:

$$
\begin{aligned}
& x_{2, t}=\alpha_{0}+\sum_{j=0}^{k_{x}} \alpha_{1, j} x_{1, t-j}+\sum_{l=0}^{k_{x}} \alpha_{2, l} x_{2, t-l}+\epsilon_{x, t}, \\
& y_{2, t}=\beta_{0}+\sum_{j=0}^{k_{y}} \beta_{1, j} y_{1, t-j}+\sum_{l=0}^{k_{y}} \beta_{2, l} y_{1, t-l}+\epsilon_{y, t},
\end{aligned}
$$

and

$$
z_{2, t}=\gamma_{0}+\sum_{i=0}^{k} \gamma_{1, i} d_{t-i}+\sum_{j=0}^{k} \gamma_{2, j} z_{1, t-j}+\sum_{q=0}^{k} \gamma_{3, q} z_{1, t-q} d_{t-q}+\epsilon_{t}
$$

where $\epsilon_{x, t}, \epsilon_{y, t}$ and $\epsilon_{t}$ are i.i.d innovations. The hypotheses also expand to test $\gamma_{3,0}=\gamma_{3,1}=$ $\ldots=\gamma_{3, k}=0$ jointly. The main purpose of introducing the lagged variables is to emphasize the dynamic structure in which the present asset returns are not only determined by the present quantities of another market, but are also affected by its previous values. One can 
further extend this framework by considering autoregressive conditional heteroscedastic $(\mathrm{ARCH})$ errors. Then the estimation and testing procedure should be adjusted; see, for example, Koenker and Zhao (1996). We leave this generalization for the future research.

\section{Quantile Regression Model}

Both (2.3) and (2.4) aim at describing the relationship between the stock returns based on the conditional mean process. However, considering the fact that time series data might exhibit different behavior across quantiles, the methods described in Section 2 cannot provide complete information on the data distribution, and they fail to capture heterogeneity across quantiles. More specifically, the relationship between the two stock returns may remain the same during the non-crisis and crisis periods on average but vary at some quantiles. To model such heterogeneity, we consider the quantile version of (2.3)

$$
Q_{z 2, t}\left(\tau \mid x_{t}\right)=\gamma_{0}(\tau)+\gamma_{1}(\tau) d_{t}+\gamma_{2}(\tau) z_{1, t}+\gamma_{3}(\tau) z_{1, t} d_{t} .
$$

where $Q_{z 2, t}\left(\tau \mid x_{t}\right)$ denotes the $\tau$-conditional quantile function of country 2's asset returns, $\tau \in(0,1)$ is a fixed value and $x_{t}=\left(1, d_{t}, z_{1, t}, z_{1, t} d_{t}\right)$. By letting $\tau$ vary from 0 to 1 continuously, we can trace out the conditional distribution of country 2's asset returns given the information on country 1's asset returns. Model (2.3) allows the interaction between the two countries' asset returns to differ over quantiles, and thus the contagion test based on quantile regression (2.3) can examine the occurrence of contagion at different quantiles.

For example, a significant $\gamma_{3}$ at lower quantiles means that the association between the two countries' markets is enhanced when country 2's market is in a decline.

More importantly, the estimated coefficient of the quantile regression (3.1) is related 
to quantile correlation, which can be considered a quantile version of the mean correlation. To demonstrate this, we start with the definition of "quantile uncorrelation" recently proposed by Komarova et al. (2012). Extending Komarova et al. (2012), quantile uncorrelation between two $l$-dimensional random vectors $y$ and $x$ can be defined as

$$
M(c y, x)=0 \quad \text { for all } \quad c \in \mathcal{R}^{l},
$$

where

$$
M(c y, x):=\arg \min _{\beta} E \rho_{\tau}\left[c y-x^{\prime} \beta-\operatorname{Quant}\left(c y-x^{\prime} \beta\right)\right]
$$

and Quant $\left(c y-x^{\prime} \beta\right):=\inf \left\{u: P\left(c y-x^{\prime} \beta \geq u\right) \geq \tau\right\}$ for a given $\tau$. If we consider the conditional quantile function of $y$ in a bivariate regression model with i.i.d. errors

$$
Q_{y}(\tau \mid x)=\beta_{0}+x^{\prime} \beta_{1}+F_{u}^{-1}(\tau),
$$

the solution to the optimization problem

$$
\left\{\hat{\alpha}(\tau), \hat{\beta}_{1}(\tau)\right\}=\arg \min _{\alpha, \beta} E \rho_{\tau}\left(y-\alpha-x^{\prime} \beta\right)
$$

estimates the population parameters $\left(\beta_{0}+F_{u}^{-1}(\tau), \beta_{1}\right)$ for a given $\tau$. Note that $\hat{\beta}_{1}(\tau)$ is equivalent to $M(c y, x)$ in the sense that $\hat{\beta}_{1}(\tau)=0$ implies $M(c y, x)=0$ because of the invariance property. Thus, we may say that the estimated coefficient of the quantile regression is an indicator of quantile correlation (but not a direct measure).

Next, we examine how the quantile regression coefficient is related to the quantile correlation. Komarova et al. (2012) used the $\mathcal{L}_{1}$ analogue of $R^{2}$ as a measure of median correlation. It takes a value of zero when $y$ is median uncorrelated with $x$. Extending the median correlation, we follow Koneker and Machado (1999) and measure the quantile correlation using

$$
\text { quanrsq }(y, x):=1-\frac{\min _{\beta} E \rho_{\tau}\left(y-x^{\prime} \beta-F_{\hat{u}}^{-1}\right)}{\min _{\beta} E \rho_{\tau}\left(y-F_{\tilde{u}}^{-1}\right)},
$$


where $\hat{u}=y-x^{\prime} \beta$ and $\tilde{u}=y$. According to Theorem 3.3 in Komarova et al. (2012) if $\beta=0$ then quanrsq $(y, x)=0$. Hence, the regression quantile reflects the degree of quantile correlation even though it is a direct measure. In our case, if we find that $\gamma_{3}(\tau)=0$ for a given $\tau$, namely $\alpha_{1}(\tau)=\beta_{1}(\tau)$, then we can infer that the quantile correlations of the two countries' stock returns are similar, and there is thus no quantile contagion.

The conditional quantile function $Q_{z 2, t}\left(\tau \mid x_{t}\right)$ can be estimated by $\hat{\gamma}(\tau)=\left[\hat{\gamma}_{0}, \hat{\gamma}_{1}(\tau), \hat{\gamma}_{2}(\tau), \hat{\gamma}_{3}(\tau)\right]$ which solves the following minimization problem

$$
\min _{\gamma \in \mathbb{R}^{4}} \sum_{t=1}^{T} \rho_{\tau}\left[z_{2, t}-\gamma_{0}(\tau)-\gamma_{1}(\tau) d_{t}-\gamma_{2}(\tau) z_{1, t}-\gamma_{3}(\tau) z_{1, t} d_{t}\right],
$$

where $\rho_{\tau}\left(\eta_{t}\right)=\eta_{t}\left[\tau-I\left(\eta_{t}<0\right)\right]$ and $I\left(\eta_{t}<0\right)$ is an indicator function (Koenker and Bassett, 1978). This is equivalent to maximizing the log-likelihood function (ML) of (2.3)

$$
\ln L(\gamma, \tau)=T \ln \tau(1-\tau)-\sum_{t=1}^{T} \rho_{\tau}\left(z_{2, t}-\gamma x_{t}\right),
$$

assuming that $\eta_{t}$ follows the asymmetric Laplace density (ALD)

$$
f\left(\eta_{t}, \sigma, \gamma\right)=\frac{1}{\sigma} \tau(1-\tau) \exp \left[-\frac{1}{\sigma} \rho_{\tau}\left(z_{2, t}-\gamma x_{t}\right)\right],
$$

with $\sigma=1$ and fixed $\tau \in(0,1)$.

In this likelihood framework, we can conveniently derive the score tests for quantile regression. Moreover, the likelihood approach enables us to consider the quasi maximum likelihood estimation (QMLE), which leads to robust estimates under distributional misspecification. Bera et al. (2008) showed that the quasi-maximum likelihood estimator $\hat{\gamma}$ converges to the normal distribution as

$$
\sqrt{T}\left(\hat{\gamma}-\gamma_{0}\right) \stackrel{d}{\rightarrow} N\left(0, V_{2}^{-1} V_{1} V_{2}^{-1}\right)
$$

where

$$
V_{1}:=E\left[\frac{1}{T}\left(\frac{\partial \ln L(\gamma, \tau)}{\partial \gamma}\right) \cdot\left(\frac{\partial \ln L(\gamma, \tau)}{\partial \gamma}\right)^{\prime}\right]_{\gamma=\gamma_{0}}, \quad V_{2}:=\left.\frac{\partial^{2} E[1 / T \ln L(\gamma, \tau)]}{\partial \gamma \partial \gamma^{\prime}}\right|_{\gamma=\gamma_{0}} .
$$


The expectations in $V_{1}$ and $V_{2}$ are taken with the true density function.

\section{Quantile Symmetric Test}

We first test the coefficient of the quantile regression with the two-sided alternative hypothesis, that is, $H_{0}: \gamma_{3}=0$ against $H_{A}: \gamma_{3} \neq 0$, and we call this the quantile symmetric test $(Q S T)$. We use Rao's score test based on the likelihood function (3.3) because it is not affected even if parameters might lie on the boundary of the maintained hypothesis. In addition, it is computationally easier than the Wald and likelihood ratio tests in many cases since it only requires the restricted estimator under the null hypothesis. We start with four necessary assumptions:

A1: $f\left(\eta_{t}, \gamma\right)$ is measurable in $\eta_{t}$ for every $\gamma$ in a compact set $\Gamma$ and continuous in $\gamma$ for every $\eta_{t}$.

A2: $\left|\log f\left(\eta_{t}, \gamma\right)\right| \leq m\left(\eta_{t}\right)$ for every $\gamma$ where $m\left(\eta_{t}\right)$ is integrable with respect to the true density distribution $G\left(\eta_{t}\right)$.

A3: $\tilde{J}:=\frac{1}{T} \sum\left[\partial \ln f\left(\eta_{t}, \gamma\right) / \partial \gamma\right] \cdot\left[\partial \ln f\left(\eta_{t}, \gamma\right) / \partial \gamma\right]^{\prime}$ and $\tilde{K}:=\frac{1}{T} \sum-\partial^{2} \ln f\left(\eta_{t}, \gamma\right) / \partial \gamma \partial \gamma^{\prime}$ exist and are continuous in $\gamma$ and $E\left(\sup _{\gamma}|\tilde{J}|\right)<\infty, E\left(\sup _{\gamma}|\tilde{K}|\right)<\infty$.

A4: The Kullback-Leibler information criterion (KLIC), $I(g, f, \gamma):=E\left(\log \left[g\left(\eta_{t}\right) / f\left(\eta_{t}, \gamma\right)\right]\right)$, reaches its unique minimum $\gamma^{*}$ interior to the parameter space $\Gamma$.

A1 ensures the existence of QMLE and a well-defined KLIC is guaranteed by A2. Both $f\left(\eta_{t}, \gamma\right)$ and $\partial \log f\left(\eta_{t}, \gamma\right) / \partial \gamma$ are continuous and differentiable except at the points where $\eta_{t}=0$; however, they have directional derivatives in all directions at these points (Koenker 
(2005) pp. 32-33). Therefore, A3 is satisfied to allow the application of the uniform law of large numbers to $J$ and $K$.

It is well known that when the true data generating process (DGP) deviates from the assumed distribution that we assume, the standard Rao's score statistic $R S=s(\tilde{\gamma})^{\prime} K(\tilde{\gamma})^{-1} s(\tilde{\gamma})$ is not valid because a distribution misspecification would lead to breaks in information matrix equality, that is, $J\left(\gamma_{g}\right) \neq K\left(\gamma_{g}\right)$, where

$$
J\left(\gamma_{g}\right)=E_{g}\left[\frac{\partial \ln f(\gamma)}{\partial \gamma} \cdot \frac{\partial \ln f(\gamma)}{\partial \gamma^{\prime}}\right] \quad \text { and } \quad K\left(\gamma_{g}\right)=E_{g}\left[-\frac{\partial^{2} \ln f(\gamma)}{\partial \gamma \partial \gamma^{\prime}}\right] .
$$

White (1982) suggested modifying the standard Rao's score test with asymptotic robust variance to ensure proper size. Then, under A1-A4, the score test under misspecification is

$$
R S^{*}=\frac{1}{T} s^{\prime}(\tilde{\gamma})^{\prime} K(\tilde{\gamma})^{-1} H(\tilde{\gamma})\left[H(\tilde{\gamma})^{\prime} V(\tilde{\gamma}) H(\tilde{\gamma})\right]^{-1} H(\tilde{\gamma})^{\prime} K(\tilde{\gamma})^{-1} s^{\prime}(\tilde{\gamma}),
$$

where $\tilde{\gamma}$ is the restricted estimator under the null hypothesis, $H(\tilde{\gamma})=\partial h(\gamma) / \gamma^{\prime}$ where $h(\gamma)$ denotes the restrictions, and $V(\tilde{\gamma})=K(\tilde{\gamma})^{-1} J(\tilde{\gamma}) K(\tilde{\gamma})^{-1}$. The modified score test $R S^{*}$ is asymptotically distributed as $\chi_{q}^{2}$ under $H_{0}$ even in presence of misspecification, where $q$ is the number of restrictions.

To test $H_{0}: \gamma_{3}=0$ against $H_{A}: \gamma_{3} \neq 0$, we partition the parameter vector $\gamma^{\prime}$ as $\left(\gamma_{A}, \gamma_{B}\right)^{\prime}$, where $\gamma_{A}=\left(\gamma_{0}, \gamma_{1}, \gamma_{2}\right)$ is the nuisance parameters and $\gamma_{B}=\gamma_{3}$ is the parameter of interest. Under the null hypothesis, the QMLE for $\gamma$ is $\tilde{\gamma}=\left(\tilde{\gamma}_{A}, 0\right)^{\prime}$.

Using the likelihood function (3.3), the score vector, $J(\gamma)$, and $K(\gamma)$ under the null hypothesis can be obtained consistent with the partition by

$$
\begin{gathered}
s(\tilde{\gamma})=\left(\mathbf{0}, s_{B}(\tilde{\gamma})^{\prime}\right)=\left(\mathbf{0}, \sum_{t=1}^{T}\left(\frac{1}{2} \operatorname{sgn}\left(\xi_{t}\right)+\tau-\frac{1}{2}\right) z_{1, t} d_{t}\right), \\
J\left(\tilde{\gamma}_{g}\right)=\tau(1-\tau) E^{*}\left[x^{\prime} x\right], \quad K\left(\tilde{\gamma}_{g}\right)=E^{*}\left[g(\xi) x^{\prime} x\right],
\end{gathered}
$$


where $\xi_{t}=z_{2, t}-\tilde{\gamma}_{0}-\tilde{\gamma}_{1} d_{t}-\tilde{\gamma}_{2} z_{1, t}$ denotes the residual in the restricted model, $E^{*}[s]=$ $\lim _{t \rightarrow \infty} \frac{1}{T} \sum_{t=1}^{T} s_{t}$ and $x_{t}=\left(1, d_{t}, z_{t}, z_{t} d_{t}\right)$. The restriction $h(\gamma)=\gamma_{3}$ leads to $H(\tilde{\gamma})=\partial h(\gamma) / \gamma^{\prime}=$ $(0,0,0,1)$.

For notational simplicity, we drop the arguments and let ' $\sim$ 'denote the quantity evaluated at $\gamma=\tilde{\gamma}$; then

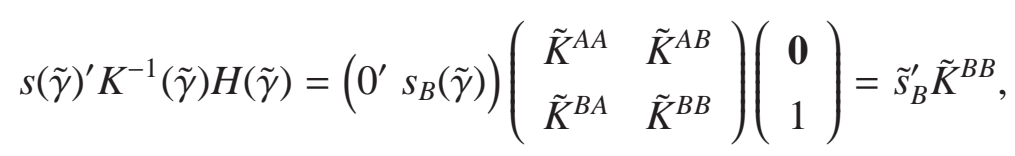

and the sandwich variance $\tilde{V}$ in (4.1) can be computed by

$$
\tilde{V}=\left(\begin{array}{cc}
\tilde{K}^{A A} & \tilde{K}^{A B} \\
\tilde{K}^{B A} & \tilde{K}^{B B}
\end{array}\right)\left(\begin{array}{cc}
\tilde{J}_{A A} & \tilde{J}_{A B} \\
\tilde{J}_{B A} & \tilde{J}_{B B}
\end{array}\right)\left(\begin{array}{cc}
\tilde{K}^{A A} & \tilde{K}^{A B} \\
\tilde{K}^{B A} & \tilde{K}^{B B}
\end{array}\right)
$$

where the subscript represents the partition of the matrix and the superscript denotes the corresponding element in its inverse matrix; for example

$$
\tilde{K}=\left(\begin{array}{ll}
\tilde{K}_{A A} & \tilde{K}_{A B} \\
\tilde{K}_{B A} & \tilde{K}_{B B}
\end{array}\right) \quad \text { and } \quad \tilde{K}^{-1}=\left(\begin{array}{cc}
\tilde{K}^{A A} & \tilde{K}^{A B} \\
\tilde{K}^{B A} & \tilde{K}^{B B}
\end{array}\right) .
$$

In addition, $\tilde{H}^{\prime} \tilde{V} \tilde{H}$ in (4.1) can be obtained in a similar fashion

$$
\tilde{H}^{\prime} \tilde{V} \tilde{H}=(\mathbf{0}, 1)\left(\begin{array}{cc}
\tilde{V}_{A A} & \tilde{V}_{A B} \\
\tilde{V}_{B A} & \tilde{V}_{B B}
\end{array}\right)\left(\begin{array}{l}
\mathbf{0} \\
1
\end{array}\right)=\tilde{V}_{B B} .
$$

With every parameter estimated, the symmetry quantile score test is summarized by the following proposition.

Proposition 1 Under A1-A4, the robust Rao's score test in the quantile regression is

$$
R S_{s}=\frac{1}{T} \tilde{s}_{B}^{\prime} \tilde{K}^{B B}\left(\tilde{K}^{A B} \tilde{K}^{B A} \tilde{J}_{A A}+\tilde{K}^{A B} \tilde{K}^{B B} \tilde{J}_{B A}+\tilde{K}^{B B} \tilde{K}^{B A} \tilde{J}_{A B}+\tilde{K}^{B B} \tilde{K}^{B B} \tilde{J}_{B B}\right)_{B B}^{-1} \tilde{K}^{B B} \tilde{s}_{B} .
$$

It asymptotically follows $\chi_{q}^{2}$ under the null hypothesis where $q$ is the number of restrictions, and it is invariant to the scaling transformation. 
The proof of invariance to the scaling transformation follows directly from the equivariance in quantile regression (see the Appendix). The invariance property suggests a direct test procedure for contagion simply with the usage of unscaled parameters instead of scaled ones.

\section{Quantile Contagion Test}

Since contagion is defined as $\gamma_{3}>0$ in (2.3), the one-sided nature of the alternative hypothesis should be taken into account to obtain a more appropriate test. In this section, we derive the quantile one-sided test $(Q O T)$ for testing the financial contagion.

One major difference between the one-sided and two-sided tests is their asymptotic distributions under the null hypothesis. Like the Wald and likelihood ratio tests with a one-sided alternative, the one-sided score test no longer asymptotically follows the $\chi^{2}$ distribution but the mixture of $\chi^{2}$ distribution. One advantage of the score test over the Wald and likelihood ratio tests is that its one-sided nature under the alternative hypothesis can be ignored since only the restricted model needs to be estimated under the null hypothesis. To ensure the limiting distribution of the efficient score test under the null hypothesis, an additional regularity condition should be satisfied (Gourieroux et al., 1982)

A5: There exists a non-singular matrix $J(\gamma)$ such that $\frac{1}{\sqrt{T}} s(\gamma)$ follows a normal distribution with variance $J(\gamma)$.

Given the asymptotic normal distribution of score vector $\frac{1}{\sqrt{T}} J^{-1} s(\gamma) \sim N\left(\iota, J^{-1}(\gamma)\right)$ under the sequence of hypotheses $H_{T}: \gamma_{3}=T^{-1 / 2} \iota$, the one-sided score test for $H_{0}: \gamma_{3}=0$ against $H_{A}: \gamma_{3}>0$ can be implemented equivalently by performing a likelihood ratio test for 
$H_{0}: \iota=T^{1 / 2} \gamma_{3}=0$ against $H_{A}: \iota=T^{1 / 2} \gamma_{3}>0$ since $\gamma_{3}>0$ is equivalent to $\iota>0$ (Silvapulle and Silvapulle, 1995). The likelihood ratio test statistic or $\bar{\chi}^{2}$-statistic for $\iota=0$ against $\iota>0$ was proposed by (Kudo, 1963)

$$
\bar{\chi}^{2}=T\left\{\bar{X}^{\prime} \Lambda^{-1} \bar{X}-\min _{\mu \geqslant 0}(\bar{X}-\mu)^{\prime} \Lambda^{-1}(\bar{X}-\mu)\right\}
$$

where $\bar{X}=T^{-1 / 2} \Lambda^{-1} d$. Here $d$ is the realization of $\iota$ with the asymptotic distribution $T^{-1 / 2} \Lambda^{-1} d \sim N\left(\iota, \Lambda^{-1}\right)$ and $\Lambda$ is the known non-singular variance matrix. With the equivalence of the likelihood ratio test and the score test, we can perform the one-sided score test with a similar procedure to that of the likelihood ratio test according to (5.1). Letting $\gamma=\left(\gamma_{A}, \gamma_{B}\right)$, the matrices $M=\frac{1}{\sqrt{T}}\left(s_{B}-K_{B A} K_{A A}^{-1} s_{A}\right)$ and $U=K^{B B} M$ evaluated at restricted MLE $\tilde{\gamma}$ can be estimated by $\tilde{M}=\frac{1}{\sqrt{T}}\left(\tilde{s}_{B}-\tilde{K}_{B A} \tilde{K}_{A A}^{-1} \tilde{s}_{A}\right)$ and $\tilde{U}=\tilde{K}{ }^{B B} \tilde{M}$. Since $\frac{1}{\sqrt{T}} J(\gamma)^{-1} s(\gamma) \sim N\left(\iota, J(\gamma)^{-1}\right)$, it follows that $M$ is also normally distributed with variance $C=J_{B B}-K_{B A} K_{A A}^{-1} J_{A B}-\left(J_{B A}-K_{B A} K_{A A}^{-1} J_{A A}\right)\left(K_{A A}^{-1}\right)^{\prime} K_{B A}^{\prime}$ under the null hypothesis. Then, for a given $\iota>0$ the asymptotic normality of $U$ under $H_{T}: \gamma_{3}=T^{-1 / 2} \iota$ can be obtained by

$$
U \stackrel{d}{\rightarrow} N\left(\iota, V_{B B}\right)
$$

With the parameters readily estimated, the one-sided score test statistic in our model is given in the following proposition

Proposition 2 Under A1-A5, the one-sided robust score test in the quantile regression can be obtained as

$$
R S_{c}=\tilde{U}^{\prime} \tilde{V}_{B B}^{-1} \tilde{U}-\inf _{b>0}\left\{(\tilde{U}-b)^{\prime} \tilde{V}_{B B}^{-1}(\tilde{U}-b)\right\}
$$

which asymptotically follows the mixture chi-square under the null hypothesis.

Since $V$ is nonsingular, symmetric, and positive definite, we can find a non-singular matrix $T$ to transform $V$ into a unit matrix-say $T V T^{\prime}=I$. By multiplying the same matrix $T$, 
$U$ converts to the uncorrelated matrix $Q=T U$, and therefore $U^{\prime} \tilde{V}_{B B}^{-1} U$ reduces to a square form $Q^{\prime} Q$. In addition, the minimum $\inf _{b>0}\left\{(\tilde{U}-b)^{\prime} \tilde{V}_{B B}^{-1}(\tilde{U}-b)\right\}$ can be thought of as the distance between the point of vector $Q$ and a cone, and thus the geometric intuition behind the statistics in (5.2) is the difference between the square length of a vector $Q^{\prime} Q$ and the distance from a point in this vector to a closed convex cone (Kudo, 1963).

The asymptotic distribution of the test statistic can be studied via the asymptotic $p$ value, which can be obtained by $\sup _{\gamma_{A}} \zeta\left(R S_{c}, V\left(\gamma_{A}\right), \mathscr{C}\right)$, where $\mathscr{C}$ denotes the set of positive real numbers and

$$
\zeta\left(R S_{c}, V\left(\gamma_{A}\right), \mathscr{C}\right)=\operatorname{Prob}\left(\left[U^{\prime} V_{B B}^{-1} U-\inf _{b \geqslant 0}\left\{(U-b)^{\prime} V_{B B}^{-1}(U-b)\right\}\right] \geqslant R S_{c}\right)
$$

As an extension of the work by Perlman (1969), the asymptotic $p$-value $\sup _{\gamma_{A}} \zeta\left(R S_{c}, V\left(\gamma_{A}\right), \mathscr{C}\right)$ lies in the interval between the lower bound $0.5 \operatorname{Prob}\left(\chi_{1}^{2} \geqslant R S_{c}\right)$ and the upper bound $0.5\left[\operatorname{Prob}\left(\chi_{q-1}^{2} \geqslant R S_{c}\right)+\operatorname{Prob}\left(\chi_{q}^{2} \geqslant R S_{c}\right)\right]$, where $q$ is the dimension of the restricted vector (see also Silvapulle and Silvapulle (1995)). The statistic in (5.2) follows a mixture of chi-squared distribution as

$$
\operatorname{Prob}\left(\bar{\chi}^{2} \geqslant c\right)=\sum_{i=0}^{q} w_{i, q} \operatorname{Prob}\left(\chi_{i}^{2} \geqslant c\right),
$$

where $w_{i, q},(i=0,1, \ldots, q)$ are the nonnegative weights determined by the nuisance parameter $\gamma_{A}$. The weights can be calculated by

$$
w_{i, q}(q, V)=\sum_{|\alpha|=i} p\left\{\left(V_{\alpha^{\prime}}\right)^{-1}\right\} p\left\{V_{\alpha, \alpha^{\prime}}\right\},
$$

where the summation extends over the nonempty subsets $\alpha$ of $\{1,2, \cdots, q\}, V_{\alpha}$ is the corresponding variance matrix of normal vector $U_{i}, i \in \alpha, V_{\alpha, \alpha^{\prime}}$ is the same under the condition $U_{j}=0$ for $j \notin \alpha$, and $p\{V\}$ denotes the probability of the multivariate normal vector $U$ larger than 0 with the distribution $U \sim N(0, V)$ (Kudo, 1963). 
In the pooled regression model (2.3), $U$ is a scalar for the single constraint $\gamma_{3}=0$; therefore, $q=1$ in this model. The weights can be obtained by

$$
w_{0,1}(V)=w_{1,1}(V)=0.5 \text {. }
$$

Note that $R S_{c}(\tau)$ is a function of $\tau$ and the weights are not dependent on $\gamma_{A}$. Hence, the "sup ${ }_{\gamma_{A}}$ " in calculating the $p$-value can be ignored and the $p$-value varies for different $\tau$ as

$$
\begin{aligned}
\sup _{\gamma_{A}} \zeta\left(R S_{c}(\tau), A\left(\gamma_{A}\right), \mathscr{C}\right) & =\sup _{\gamma_{A}} \operatorname{Prob}\left(\left[U^{\prime} A_{B B}^{-1} U-\inf _{b \geqslant 0}\left\{(U-b)^{\prime} A_{B B}^{-1}(U-b)\right\}\right] \geqslant R S_{c}(\tau)\right) . \\
& =0.5 \operatorname{Prob}\left(\chi_{1}^{2} \geqslant R S_{c}(\tau)\right) .
\end{aligned}
$$

Further computation is required in the case of the dynamic model in (2.4). If we consider the model with one lagged variable

$$
z_{2, t}=\gamma_{0}+\gamma_{1,0} d_{t}+\gamma_{2,0} z_{1, t}+\gamma_{3,0} z_{1, t} d_{t}+\gamma_{1,1} d_{t-1}+\gamma_{2,1} z_{1, t-1}+\gamma_{3,1} z_{1, t-1} d_{t-1}+\epsilon_{t},
$$

we need to test the hypothesis $H_{0}: \gamma_{3,0}=0, \gamma_{3,1}=0$ with the alternative hypothesis $H_{A}: \gamma_{3,0}>0, \gamma_{3,1}>0$. Therefore, $q=2$ in the dynamic model (5.3). In this case, the weights can be calculated by

$$
\begin{aligned}
& w_{0,2}(V)=0.5 \pi^{-1}\left(\pi-\cos ^{-1} \rho_{1,2}\right), \\
& w_{1,2}(V)=0.5, \\
& w_{2,2}(V)=0.5 \pi^{-1} \cos ^{-1} \rho_{1,2} .
\end{aligned}
$$

where $\rho_{i, q}$ is given by the $(i, q)$ th element of the correlation matrix of $U$ :

$$
\left[\operatorname{diag}(U)^{-1}\right]^{-\frac{1}{2}}(U)^{-1}\left[\operatorname{diag}(U)^{-1}\right]^{-\frac{1}{2}}
$$

Different from the weights in the static model, the weights here are functions of $\gamma_{A}$, namely $w_{i, q}\left(\gamma_{A}\right)$, with the intermediate parameter $V$. Hence the $p$-value can be obtained 
by optimizing the probability with $\gamma_{A}$ carried by the weights

$$
\begin{aligned}
& \sup _{\gamma_{A}} \operatorname{Prob}\left(\left[(U)^{\prime}(V)_{B B}^{-1}(U)-\inf _{b \geqslant 0}\left\{(U-b)^{\prime}(V)_{B B}^{-1}(U-b)\right\}\right] \geqslant R S_{c}(\tau)\right) \\
= & \sup _{\gamma_{A}} \sum_{i=0}^{2} w_{i, 2}\left(\gamma_{A}\right) \operatorname{Prob}\left(\chi_{i}^{2} \geqslant R S_{c}(\tau)\right)
\end{aligned}
$$

where $\chi_{i}^{2}$ denotes the chi-square distribution with $i$ degrees of freedom.

\section{Monte Carlo Simulation}

In the simulation we mainly focus on the comparison of the proposed quantile tests and the correlation-based $F R$ tests. $F R$ tests are mostly popular for testing financial contagion because they are based on the most popular definition of contagion, are easy to implement, and have better performance in most cases. Our quantile contagion can be thought as a quantile version of correlation-based tests, and therefore this comparison is of particular interest. More comparison between contagion tests can be found in Dungey et al. $(2005 a, b)$.

\subsection{Simulation Design}

The experiment design follows the setup of Dungey et al. (2005b) in which asset returns in non-crisis and crisis structures are determined by common factors $w_{t}$ together with idiosyncratic factors $u_{t}$ (see also Pericoli and Sbracia (2003)). The common factors describe the fundamental elements that determine the average level of asset returns worldwide, while the idiosyncratic component captures the unique factor that differs from individual markets. In our experiments, we study the interrelationship of two asset markets using 
observations during tranquil and volatile periods. Stock market contagion is defined as transmission from country 1 to country 2 . We model the non-crisis linkage as

$$
\begin{aligned}
& x_{1, t}=4 w_{t}+2 u_{1, t}, \\
& x_{2, t}=3 w_{t}+4 u_{2, t},
\end{aligned}
$$

where $w_{t}=\rho w_{t-1}+u_{w, t}, u_{w, t} \sim N\left(0, h_{t}\right), h_{t}=\omega_{x}^{2}\left(1-\alpha-\beta+\alpha u_{w, t-1}^{2}+\beta h_{t-1}\right)$, and $u_{i, t} \sim G_{x}\left(u_{i, t}\right)$ for some distribution $G_{x}(\cdot)$. This setup allows for autocorrelation as well as a GARCH structure in the common factor. The parameter $\rho$ reflects the degree of autocorrelation while $\alpha$ and $\beta$ jointly control the GARCH process with time varying variance. In addition, $\omega_{x}$ determines the variance of the common factor, and it can shift during the tranquil and volatile periods to generate the common factor structural break. The crisis model is an extension of the non-crisis framework of (6.1) and (6.2) that adds country 1's idiosyncratic factor to the country 2 's asset return $y_{2, t}$.

$$
\begin{aligned}
& y_{1, t}=4 w_{t}+2 u_{1, t}, \\
& y_{2, t}=3 w_{t}+3 u_{2, t}+2 \delta u_{1, t},
\end{aligned}
$$

where $w_{t}=\rho w_{t-1}+u_{w, t}, u_{w, t} \sim N\left(0, h_{t}\right), h_{t}=\omega_{y}^{2}\left(1-\alpha-\beta+\alpha u_{w, t-1}^{2}+\beta h_{t-1}\right), u_{i, t} \sim G_{y}\left(u_{i, t}\right)$ and $\delta$ denotes the contagion strength. For the distribution of idiosyncratic factor $G_{i}, i=x, y$, three distributions are considered: (i) the normal distribution, (ii) Student's $t$ distribution with degree of freedom $3^{1}$, and (iii) Hansen (1994)'s skewed $t$ distribution.

In our experiment design, the two markets are relatively uncorrelated with independent idiosyncratic factors in the tranquil period. However, during the crisis period, country 1's idiosyncratic factor affects country 2's asset returns, increasing the correlation be-

\footnotetext{
${ }^{1}$ We also perform the experiment under Student's $t$ distribution with degrees of freedom five and seven. The results are substantially unchanged.
} 
tween the two countries. We consider various characteristics of the data, such as the GARCH process, no autocorrelation data, weak autocorrelation data, strong autocorrelation data and data with a structural break. Two structural breaks are studied: (1) a structural break in the world factor $w_{t}$, which is generated by changing $\omega$, and (2) a structural break in the idiosyncratic factor, which is generated by changing the variance of the idiosyncratic factor in different periods. When $G$ is normally distributed, $u_{i, x} \sim N\left(0, \kappa_{x}^{2}\right)$ and $u_{i, y} \sim N\left(0, \kappa_{y}^{2}\right)$, the idiosyncratic structural break comes from the deviation between $\kappa_{x}$ and $\kappa_{y}$. When $G$ follows Student's $t$ distribution, the idiosyncratic break is generated by changing the degree of freedom. For the skewed $t$ distribution, we use Hansen (1994)'s generalized $t$ distribution (see also Jondeau and Rockinger (2003))

$$
u(m \mid v, \phi)= \begin{cases}c\left(1+\frac{1}{v-2}\left(\frac{m}{1-\phi}\right)^{2}\right)^{(v+1) / 2} & \text { if } m<-a / b, \\ c\left(1+\frac{1}{v-2}\left(\frac{m}{1+\phi}\right)^{2}\right)^{(v+1) / 2} & \text { if } m \geqslant-a / b,\end{cases}
$$

where $c \equiv \Gamma[(v+1) / 2] /[\sqrt{\pi(v-2)} \Gamma(v / 2)]$ normalizes function $u(\cdot)$ as a p.d.f. with the mean $4 \phi c(v-2) /(v-1)$ and variance $1+3 \phi^{2}-a^{2}$ (Jondeau and Rockinger, 2003). The scaling parameter $v$, interpreted as the order of moment, characterizes the height and tail of the distribution, while $\phi$ controls the skewness of the distribution. Based on (6.5), we can generate the break by changing either $\phi$ or $v$.

Although the model (6.1)-(6.4) is a typical way of generating contagion (Dungey et al., $2005 a, b)$, we can show that under some specific parameterization the mean correlation decreases as contagion strength $\delta$ increases. This can be seen in Figure 1, and a mathematical derivation is given in the Appendix. In this case, correlation-based tests would incorrectly report no contagion. Therefore, we compare our quantile contagion with $F R$ tests in two scenarios when correlation increases and when correlation decreases as contagion strengthens. In the following, we call these "increasing correlation" and "decreasing 
correlation" for short. The decreasing correlation scenario can be generated by choosing a smaller variance of the idiosyncratic factor than that of the common factor. ${ }^{2}$

\section{[Figure1, Table1]}

The whole sample period $T$ is taken by 200,500 and 1000 with sample sizes of 100 , 250 , and 500 of returns in the crisis period respectively. We let $\delta$ vary in a range from 0 to 12 , and each experiment is duplicated 3000 times. The parameterization in both the increasing and decreasing correlation cases is given in Table 1.

\subsection{Size Property}

Tables 2 and 3 report the size of the $Q S T, Q O T$, and FR tests in the increasing and decreasing correlation cases. Each of the experiments are based on critical values of $5 \%$. We first consider the increasing correlation case. In this case, $F R_{1}$ is always undersized in all experiments, which implies that the null hypothesis of no contagion is over accepted. The poor size property of $F R_{1}$ may be due to the inappropriate assumption of independence between the non-crisis and crisis samples in its asymptotic variance. Thus, we focus on comparing $Q S T$ and $Q O T$ with $F R_{2}$ and $F R_{3}$ in the following. $F R_{2}$ and $F R_{3}$ are competitive with $Q S T$ and $Q O T$ in the normal distribution case, but not in the Student's $t$ and skewed $t$ cases. The four test statistics exhibit good size properties in most experiments of the normal distribution except for Experiments I and IV. We note that oversize of all the tests in Experiment IV of common the structural break may be because an increase in the volatility of the common factor leads to a higher degree of similarity between the two

\footnotetext{
${ }^{2}$ The GARCH experiments with decreasing correlation are implemented by generating an idiosyncratic factor with a smaller amplitude than the common factor.
} 
markets even though the contagion does not occur. Under the Student's $t$ distribution, $F R_{2}$ yields relatively good size performance in most experiments except for Experiments IV and V (structure break), and the size of $F R_{3}$ is inflated in most cases. Compared to the $F R$ tests, $Q S T$ and $Q O T$ maintain better size conduction in most of the experiments. The results under the skewed $t$ distribution are very similar to the Student's $t$ case in that $Q S T$ and $Q O T$ generally beat the $F R$ tests. We thus conclude from the above results that $Q S T$ and $Q O T$ perform better than all forms of $F R$ in terms of size when the data distribution is generated with fatter tail and skewness.

Although the size of $F R_{2}$ and $F R_{3}$ is comparable to $Q S T$ and $Q O T$ in the increasing correlation case, it is heavily dominated by our quantile tests in the decreasing correlation case, as reported in Table 3. $F R_{1}$ remains severely undersized for all experiments. $F R_{2}$ and $F R_{3}$ have an undesirable size property in most of the experiments when we have decreasing correlation. For example, under the normal distribution the size of $F R_{2}$ is less than 0.020 in all experiments, and $F R_{3}$ has an oversized value in Experiments I, II and III. Under the skewed $t$ distribution, $F R_{2}$ and $F R_{3}$ tend to be oversized in most experiments. In contrast, $Q S T$ and $Q O T$ report much better size properties for all experiments. For most experiments, the best size performance of $Q O T$ occurs for $\tau \in(0.3,0.7)$ except Experiment IV and V.

[Table2, Table3]

\subsection{Power Property}

The finite sample power property, which reflects the efficiency of contagion detection, is reported in Figures 2-4. Each of the experiments are based on critical values of 5\%. 
We consider the increasing correlation case. The highest power property of $Q O T$ appears in the median (0.5 quantile), and the power decreases when the quantile goes to two extremes. $F R_{2}$ and $F R_{3}$ show a good power property, which is consistent with the results in Dungey et al. (2005b). Among the three forms of $F R$ tests, $F R_{2}$ exhibits the best power property in all experiments, and $F R_{2}$ even beats the quantile tests the under the normal distribution case (Figure 2). This shows the importance of correctly specifying that the non-crisis and crisis periods should be separated to satisfy the independence. Comparing the power of the $F R$ tests to that of $Q O T$ at the 0.5 quantile, we see that $Q O T$ is superior to $F R_{1}$ and $F R_{3}$ but not to $F R_{2}$ in the normal case. However, when we consider the Student's $t$ and skewed $t$ cases where the data are characterized by a fatter tail and higher peak, quantile tests are shown to have better power than $F R$ tests. Since financial data are mostly non-normally distributed, these cases are of more practical interest. For example, under Student's $t$ distribution $Q O T$ beats all $F R$ tests in all experiments.

We next compare the tests in the decreasing correlation case. In this case the quantile tests show particular superiority. All forms of the $F R$ tests fail to capture the contagion when the contagion strength $\delta$ rises (Figures 2-4), and none of their powers are monotonically increasing with $\delta$. For example, $F R_{1}$ and $F R_{2}$ diminish to zero when $\delta$ exceeds 8 in Experiments I, II, III, and IV under the normal distribution (Figure 2). In contrast, the power of $Q O T$ remains monotonically increasing, although it is less powerful than in the increasing correlation case. Under the Student's $t$ distribution, the $F R$ tests behave differently from the normal cases (Figure 3). When $\delta$ increases from 0 to 2 , the $F R$ tests have increasing power, but the power drops when $\delta$ exceeds 2. Similar behavior of the $F R$ tests is found under the skewed $t$ distribution. However, in all cases $Q O T$ produces monotonically increasing power as $\delta$ increases. 


\section{[Figure2, Figure3, Figure4]}

Based on the above comparison, we conclude that the proposed quantile tests ( $Q S T$ and $Q O T)$ generally produce better size and power properties than $F R$ tests, especially when the data are skewed and leptokurtic. Moreover, correlation-based tests may fail to capture contagion when it occurs not in the mean, and our quantile contagion test $Q O T$ works well and is therefore particularly useful in this case. In this sense, $Q O T$ is more robust than the $F R$ tests.

\section{Empirical Analysis}

We evaluate our quantile contagion tests and correlation-based $F R$ tests using three real data sets. We reexamine whether contagion existed in the 1994 Tequila crisis, the 1997 Asian crisis, and the 2001 Argentine crisis.

Before Forbes and Rigobon (2002) the literature using correlation-based tests had generally concluded that cross-market correlation increased significantly during crises, and that there was contagion in these crises. Forbes and Rigobon (2002) pointed out that previous correlation-based tests were upward biased and inaccurate because of heteroscedasticity, and they proposed adjusted tests ( $F R$ tests) to overcome spurious contagion problems. They found that only a small proportion of transmission channels were significant in the 1987 U.S. market crash, the 1994 Tequila crisis, and the 1997 Asian crisis. Thus they found no strong evidence of contagion in these crises, only interdependence. However, we argue that the results of the $F R$ tests should be interpreted with caution since they only indicate the relationship at an average level. We will compare the quantile contagion with 
the $F R$ tests.

The simulation analysis has shown that among the three forms of $F R$ tests $F R_{2}$ generally possesses the best finite sample properties. ${ }^{3}$ Therefore our empirical analysis will focus on comparing $F R_{2}$ and quantile contagion test $Q O T$. The stock market returns of each country are calculated as rolling-average two-day returns based on the aggregate stock market index. ${ }^{4}$ All the test statistics are considered under a bivariate framework; that is, we only consider the transmission of volatility between two countries. Before we evaluate all the tests, we take the preliminary step of extracting the residual from the bivariate VAR process for the two countries with one lag over the full sample periods to exclude any common factor in the data. We perform our proposed tests, $Q O T$ for $\tau \in[0.05,0.95]$, and the significance level is set at $5 \%$ for all cases.

\subsection{Asian Crisis}

In June 1997 a financial crisis broke out in Asia, causing great devastation in Asia's financial markets. The Thai baht has experienced substantial depreciation following the Thai government's proposal of a floating exchange rate policy on June 2, 1997. At first, only certain southeastern Asian countries, such as Malaysia and Indonesia, appeared to be affected. However, after Hong Kong's market crashed in mid-October, other Asian countries and even some Western countries appeared to be involved in this crisis. Thus, we include the stock returns of nine countries in this analysis: Japan, South Korea, Malaysia, Thailand, Hong Kong, the Unite States, Germany, the Unite Kingdom, and Australia. The indices cover a span of one year and eight months from January 1, 1997 to August 31,

\footnotetext{
${ }^{3} F R_{2}$ and $F R_{3}$ are largely similar, and $F R_{2}$ beats $F R_{3}$ in most cases.

${ }^{4}$ The daily returns are also adjusted for weekends and holidays
} 
1998 with a sample size of $436 . .^{5}$ Figure 5 provides time series plots of the stock return rates of some countries in the Asian crisis, from which we note an obvious increase in the volatility of the return rates after October 20, 1997. Accordingly, the entire sample is divided into a tranquil period from January 1, 1997 to October 19, 1997 and a turmoil period from October 20, 1997 to August 31, 1998.

\section{[Figure 5]}

Table 4 presents the descriptive statistics and some preliminary tests. All the series are asymmetrically distributed: the returns of Hong Kong, South Korea, Malaysia, Japan, and Thailand are positively skewed, while the returns of the Unite States, the Unite Kingdom, Australia, and Germany are negatively skewed. Some series show excess kurtosis, including Hong Kong, Malaysia, the Unite States, and Australia, indicating a higher probability of the existence of extreme-valued returns. The non-normal characteristics are confirmed by the Jarque-Bera test. The results of the Ljung-Box test indicate that the stock returns of Hong Kong, South Korea, Japan, the Unite Kingdom, Germany, and Thailand exhibit significant autocorrelations with lag $=5$. The ARCH LM test shows that all the countries except Malaysia have significant ARCH effects with lag $=5$. These observations suggest that the real data are highly non-normal, and therefore, a simulation design with excess skewness and kurtosis, autocorrelation, and ARCH effects is of more practical interest.

\section{[Table 4]}

Tables 5 and 6 report the intercorrelation among the nine stock markets, and we can see that the intercorrelation increases significantly in the crisis period except for between

\footnotetext{
${ }^{5}$ The data exclude the holidays and missing values
} 
Hong Kong and South Korea. Therefore, if we only consider intercorrelation, we would tend to conclude the existence of contagion in the Asian crisis. However, Forbes and Rigobon (2002) pointed out that the intercorrelation is conditional, and upward biased. They used adjusted tests based on unconditional correlation and reached the opposite conclusion of no contagion.

\section{[Table 5, Table 6]}

In the following, we compare our tests with $F R_{2}$ and examine the contagion effect within and outside of Asian countries.

\subsubsection{Within Asia}

Figure 6 shows representative transmission channels within Asia between Thailand and Malaysia, Thailand and Hong Kong, Japan and South Korea, and Japan and Malaysia. Channels related with Thailand are of interest because it is considered to be the origin of the Asian crisis, and channels related with Japan are considered since it plays a crucial role in the Asian economy. More results are available upon request. The graphs in the upper row give the values of test statistics for $\tau \in[0.05,0.95]$. Since $F R_{2}$ does not change over $\tau$, its curve is a straight line in the graph. The graphs in the bottom row give the $p$-values of $Q O T$. The quantile contagion test rejects the null hypothesis of no contagion if the $p$-values of $Q O T$ are under the $5 \%$ line, while the correlation-based test $F R_{2}$ rejects the null hypothesis when its value is greater than 1.644 , the critical value of the one-sided $t$-statistic at size 5\%. Since we perform all the tests in a bivariate framework, we test the channels from country 1 to country 2 as well as those from 2 to 1 .

We first investigate the channels related with Thailand. QOT reports significant im- 
pacts from Thailand to Malaysia and to Hong Kong at almost all quantiles. This is in line with the results of the $F R_{2}$ test. However, the results of the two tests differ in the reverse transmission. $F R_{2}$ shows that the contagion from Malaysia to Thailand and from Hong Kong to Thailand is weakly significant $\left(F R_{2} \approx 1.69\right.$ and $F R_{2} \approx 1.80$, respectively), while $Q O T$ suggests that the reverse transmission is significant at some specific quantiles. More precisely, QOT shows that the transmission from Malaysia to Thailand is significant at quantiles of $0.15,0.19,0.2,0.25,[0.47,0.86]$, and $[0.91,0.95]$; the transmission from Hong Kong to Thailand is significant at quantiles of $[0.14,0.67], 0.69,[0.73,0.76]$, and $[0.84,0.91]$. These results suggest that Thailand's stock returns are affected by the Malaysian and Hong Kong markets when they are in a decline (at lower quantiles). We note that $Q O T$ does not conflict with $F R_{2}$ in the sense that $Q O T$ also finds contagion around the median and mean levels in these transmission channels. However, QOT provides information on the whole distribution, not only the mean as reported by $F R_{2}$. Similar results are found in the transmission channels between Thailand and Korea, and between Thailand and Japan.

Next, we examine the channels related with Japan. $F R_{2}$ indicates no contagion from Japan to South Korea $\left(F R_{2} \approx 0.63\right)$. QOT confirms that this channel is indeed not significant at most quantiles, but it is significant at quantiles from 0.50 to 0.79 . This implies that Japan's stock returns are relatively unrelated with those of South Korea, but when the South Korean stock market is in an advance it is significantly correlated with the Japanese market. For the reverse channel from South Korea to Japan, $Q O T$ and $F R_{2}$ both report no significant contagion. $F R_{2}$ and $Q O T$ also differ in the transmission channels between Japan and Malaysia. $F R_{2}$ reports no contagion from Japan to Malaysia $\left(F R_{2} \approx 1.36\right)$ or in the reverse channel $\left(F R_{2} \approx 0.67\right)$. $Q O T$ suggests that contagion from Japan to Malaysia 
exists at quantiles from 0.19 to 0.94 and in the reverse channel at some lower quantiles $(<0.22)$ and upper quantiles $(>0.77)$. The influence from Japan to Malaysia may be due to Japan's large investment in Malaysia, but the Malaysian stock market hardly affects Japan except in some extreme cases.

\section{[Figure 6]}

\subsubsection{From Asia to the West}

The 1997 Asian crisis also affected the Unite States, Australia, and some European countries. Since Hong Kong and Japan may be the most important financial centers in Asia, and since they have strong connections with these Western countries, we focus on the channels related with Hong Kong, Japan, and some Western countries. We present the results for Japan and the Unite States, Japan and Australia, Hong Kong and the Unite States, and Hong Kong and the Unite Kingdom in Figure 7.

We first consider the channels related with Japan. $F R_{2}$ reports no contagion from Japan to the Unite States $\left(F R_{2} \approx 1.58\right)$, while $Q O T$ suggests significant contagion at some lower quantiles, 0.07 and $[0.1,0.44]$, and at quantiles slightly above the median, $0.65,0.67$, and $[0.77,0.81]$. The reverse impact is also not significant according to $F R_{2}\left(F R_{2} \approx 1.55\right)$, but $Q O T$ finds it to be prominent at some lower quantiles, $0.06,[0.08,0.22]$, and $[0.27,0.52]$. This implies that the connection between Japan and the Unite States generally increases when either of the two markets suffers from a sharp drop in the crisis period. For channels from Japan and Australia, both $F R_{2}$ and $Q O T$ find significant contagion. However, $F R_{2}$ detects no contagion in the reverse channel, and $Q O T$ finds contagion at quantiles $[0.28,0.6]$ and $[0.72,0.79]$. 
Next, we study the channels between Hong Kong and the Western countries. $F R_{2}$ shows no contagion from Hong Kong to the Unite States $\left(F R_{2}=0.86\right)$, while $Q O T$ finds it to be significant at some upper quantiles from 0.69 to 0.94 . Both tests suggest a significant influence in the reverse channel. This suggests that the U.S. market is more closely related with Hong Kong's market when it is in an advance and that the Hong Kong's market is significantly affected by the U.S. market most of the times. This is evidence that Hong Kong is an international financial center and that its market is easily influenced by some international markets, especially large ones. For the relation between Hong Kong and the Unite Kingdom, we find similar results. From Hong Kong to the Unite Kingdom, $F R_{2}$ again reports no contagion $\left(F R_{2}=1.111\right)$, but $Q O T$ detects significant contagion at some lower $(<0.24)$ and upper quantiles $(>0.56)$. From the Unite Kingdom to Hong Kong, both tests find significant results. The associations between Hong Kong and Australia, and between Hong Kong and Germany are largely similar. Contagion from Hong Kong to these countries is detected by $Q O T$ at some quantiles but not by $F R_{2}$, while the reverse transmission is shown to be significant by both tests.

We note from the empirics that if $F R_{2}$ finds significant contagion then $Q O T$ reports significant results at most quantiles. However, when $F R_{2}$ fails to detect any contagion $Q O T$ may report significance at a few quantiles. In general, $Q O T$ can detect contagion that $F R_{2}$ ignores, especially at lower and upper quantiles, and it does not miss significant results if $F R_{2}$ also detects them.

\section{[Figure 7]}




\subsection{Tequila Crisis}

In December 1994, an economic crisis began in Mexico following by the sharp decline of the Mexican peso. Subsequently, several South American countries fell into the crisis period, and their stock indices experienced a sudden decline. We examine the contagion effects between the Unite States, Chile, Mexico, Argentina, and Venezuela. These four South American countries particularly suffered from this crisis, and the Unite States is included because of its close connections with these four countries. The indices span a period of nine months from June 1, 1994 to March 2, 1995, with 198 sample points. The change point from the non-crisis period to the crisis period is December 12, 1994.

We also make a preliminary analysis of the characteristics of the data, and we find that all series are skewed and leptokurtic, and therefore non-normally distributed. The LjungBox and ARCH tests show that the stock returns of all South American countries are autocorrelated with ARCH effects. The correlations between Chile and Argentina, Mexico and Chile, Venezuela and Chile, and Argentina and Mexico increase significantly in the crisis period. However, the correlations between Chile and the Unite States, Argentina and the Unite States, Argentina and Venezuela, Mexico and the Unite States, Mexico and Venezuela, the Unite States and Venezuela decrease in the crisis period. The results of this preliminary analysis are available upon request.

Figure 8 presents the results of the two tests for the channels between the Unite States and Mexico, Mexico and Argentina, Mexico and Chile, and Chile and Argentina. We first examine the association between the Unite States and Mexico. $F R_{2}$ reports no significant impact in either direction of this channel $\left(F R_{2} \approx-0.80\right.$ from Mexico to the Unite States, and $F R_{2} \approx 0.12$ for the reverse direction). $Q O T$ is generally in line with $F R_{2}$ except that it 
detects significant effects from the Unite States to Mexico only at quantiles 0.89 and 0.9. This implies that the association between the Unite States and Mexico did not experience significant change during the crisis. Next we examine the relation between Mexico and Argentina. $F R_{2}$ reports no contagion from Mexico to Argentina $\left(F R_{2} \approx 0.10\right)$, while $Q O T$ detects contagion at quantiles $[0.37,0.74]$ and $[0.77,0.89]$. Both $F R_{2}$ and $Q O T$ find the reverse effect to be not significant $\left(F R_{2} \approx-0.14\right.$ and $Q O T$ is not significant except at quantile 0.92). Thus, the remarkable increase in the correlation between Mexico and Argentina is mainly due to the contagion effect from Mexico to Argentina. We next consider the relation between Mexico and Chile. Both $F R_{2}$ and $Q O T$ find no significant contagion from Mexico to Chile $\left(F R_{2} \approx 0.33\right.$ and $Q O T$ is significant only at quantile 0.23$)$. However, $Q O T$ indicates strong impacts from Chile to Mexico at lower quantiles $[0.09,0.15]$ and upper quantiles, $[0.78,0.79], 0.87,0.89$, and 0.92 . This effect is ignored by $F R_{2}$. This implies that Chile has a strong impact on the Mexican market when the latter experiences a sharp slump or an advance. Finally we investigate the channel between Chile and Argentina. $F R_{2}$ finds no significant results in either direction. $Q O T$ indicates a significant effect from Chile to Argentina at quantiles $[0.11,0.86]$ and $[0.88,0.95]$, but not a reverse effect. Most of the other channels are shown to be not significant by both $F R_{2}$ and $Q O T$.

\section{[Figure 8]}

Based on the above analysis, we conclude that Mexico and Chile were the two main sources of contagion in the Tequila crisis, and they had impacts on other South American countries to various extents. However, these effects are generally not captured by the correlation-based test $F R_{2}$. We also note that this crisis did not spread to the Unite States to a significant degree. In contrast to the Asian crisis, we observe that the correlations be- 
tween some South America countries decreased in the Tequila crisis, leading to negative values of the $F R_{2}$ test. In this case, $Q O T$ can still capture contagion at some extreme quantiles. This again shows the difference between the mean correlation and the correlation at quantiles.

\subsection{Argentine Crisis}

Finally we test the contagion effect in the Argentine crisis, which began in 2001. In that crisis, some South American countries, including Brazil, Chile, Mexico, suffered from an unfavorable balance of trade, heavy international debts, and high inflation rates. We focus on four South America countries, Argentina, Mexico, Brazil, and Chile, that were involved in this crisis to varying extents. The entire sample period spans from July 9, 2000 to December 3, 2001, with a sample size of 367. We choose July 9, 2001 as the change point from the non-crisis period to crisis period because it is the date Argentina became involved in this crisis.

The descriptive statistics show that all series are non-normal. The stock returns of all countries have less kurtosis than the normal distribution. The Ljung-Box test indicates that all series exhibit significant autocorrelation effects with lag $=5$ except Argentina and Brazil, and the ARCH test reports significant ARCH effects for Chile and Brazil. The correlation studies suggest that the correlations of most channels increase significantly in the crisis period.

Figure 9 presents the associations between Argentina and Chile, Argentina and Brazil, Chile and Mexico, and Chile and Brazil. We first examine the channels related with Argentina. $F R_{2}$ shows that these channels are not significant in either direction. $Q O T$ finds 
similar results of no contagion in most of the channels. The only exception is the association between Argentina and Chile. $Q O T$ reports a significant effect from Argentina to Chile around the median and a significant reverse effect at lower quantiles, [0.06,0.2] and $[0.35,0.41]$. This shows that the Argentine market had a strong impact on the Chilean market when the latter was under a normal situation (around the median), but Chile affected Argentina only when the latter was in a bear market (lower quantile).

We next consider the channels related with Chile. QOT finds the impact from Mexico to Chile to be significant at lower quantile [0.05,0.32], while $F R_{2}$ ignores this effect $\left(F R_{2} \approx 0.17\right)$. Both tests report insignificant feedback from Chile to Mexico. Both tests find strong contagion between Brazil and Chile in both directions. More particularly, according to QOT the Brazilian market affects the Chilean market significantly at nearly all quantiles, and this is confirmed by $F R_{2}\left(F R_{2} \approx 3.05\right)$. QOT shows that the reverse impact is significant at quantiles $[0.17,0.21],[0.42,0.72]$, and $[0.9,0.95]$, while $F R_{2} \approx 2.14$ also detects contagion.

\section{[Figure 9]}

In summary, there is significant contagion between Brazil and Chile in the Argentine crisis, which is captured by both tests. However, the contagion between Argentina and Chile and between Mexico and Chile is captured by QOT at some quantiles, but not by $F R_{2}$. Except for these channels, there is no strong evidence for the spread of the Argentine crisis, at least within our samples. 


\section{References}

K.-H. Bae, G. A. Karolyi, and R. M. Stulz. A new approach to measuring financial contagion. Review of Financial Studies, 16(3):717-763, 2003.

A. K. Bera, G. V. Montes-Rojas, and S. Y. Park. Which quantile is the most informative? quantile-mode regression. Working Paper, 2008.

L. Cappiello, B. Gerard, and S. Manganelli. The contagion box: Measuring financial market co-movements by regression quantiles. working paper, 2004.

M. Dungey, R. Fry, B. Gonzlćlez-Hermosillo, and V. L. Martin. Empirical modelling of contagion: A review of methodologies. Quantitative Finance, 5(1):9-24, 2005a.

M. Dungey, R. Fry, B. Gonzlćlez-Hermosillo, and V. L. Martin. Sampling properties of contagion tests. Working Paper, 2005b.

C. A. Faveroa and F. Giavazzi. Is the international propagation of financial shocks nonlinear? evidence from the erm. Journal of International Economics, 57(1):231-46, 2002.

K. J. Forbes and R. Rigobon. Measuring Contagion: Conceptual and Empirical Issues,, pages 44-47. Kluwer Academic Publishers, Norwell, MA, 2001.

K. J. Forbes and R. Rigobon. No contagion, only interdependence: Measuring stock market co-movements. Journal of Finance, 57(5):2223-61, 2002.

C. Gourieroux, A. Holly, and A. Monfort. Likelihood ratio test, wald test, and kuhntucker test in linear models with inequality constraints on the regression parameters. Econometrica, 50(1):63-80, 1982. 
B. E. Hansen. Autoregressive conditional density estimation. International Economic Review, 35(3):705-730, 1994.

E. Jondeau and M. Rockinger. Conditional volatility, skewness, and kurtosis: existence, persistence, and comovements. 27(10):1699-1737, 2003.

M. A. King and S. Wadhwani. Transnlission of volatility between stock markets. Review of Financial Studies, 3(1):5-33, 1990.

R. Koenker. Quantile Regression,. Cambridge University Press, Cambridge, 2005.

R. Koenker and G. Bassett. Quantile regression. Econometrica, 46(1):33-50, 1978.

T. Komarova, T. Severini, and E. Tamer. Quantile uncorrelation and instrumental regressions. Working Paper, 2010.

R. Koneker and J. A. F. Machado. Goodness of fit and related processes for quantile regression. Journal of the American Statistical Association, 94(448):1296-1310, 1999.

A. Kudo. A multivariate analogue of the one-sided test. Biometrika, 50(3/4):403-418, 1963.

S. B. Lee and K. J. Kim. Does the october 1987 crash strengthen the comovements among national stock markets? Review of Financial Studies, 3(1):89-102, 1993.

M. Pericoli and M. Sbracia. A primer on financial contagion. Journal of Economic Surveys, 17(4):571-608, 2003.

M. D. Perlman. One-sided testing problems in multivariate analysis. The Annals of Mathematical Statistics, 40(2):549-567, 1969.

H. M. Pesaran and A. Pick. Econometric issues in the analysis of contagion. Biometrika, 31(4):1245íC1277, 2007. 
J. C. Rodriguez. Measuring financial contagion: A copula approach. Journal of Empirical Finance, 14(3):401-423, 2007.

M. J. Silvapulle and P. Silvapulle. A score test against one-sided alternatives. Biometrika, 90(429):342-349, 1995.

H. White. Maximum likelihood estimation of misspecified models. Econometrica, 50(1): $1-25,1982$.

\section{Appendix}

\section{Appendix 1: Proof of the Invariance Property of the Scaling Transforma- tion}

In this appendix, we show the invariance property of the scaling transformation in quantile regression. Let

$$
\psi\left(\tau, z_{T}, X ; \gamma\right)=\sum_{t: z z_{2, t}>\gamma x_{t}} \tau\left|z_{2, t}-\gamma x_{t}\right|+\sum_{t: z 2, t<\gamma x_{t}}(1-\tau)\left|z_{2, t}-\gamma x_{t}\right| .
$$

Note that the following properties are possessed by $\psi\left(\tau, z_{T}, X ; \gamma\right)$ (Koenker and Bassett 1978): (1) $\kappa \psi\left(\tau, z_{T}, X ; \gamma\right)=\psi\left(\tau, \kappa z_{T}, X ; \kappa \gamma\right), \kappa \in[0, \infty) ;(2) \psi\left(\tau, z_{T}, X ; \gamma\right)=\psi\left(\tau, z_{T}, X A ; A^{-1} \gamma\right)$, $|A| \neq 0$; and (3) $\psi\left(\tau, z_{T}, X ; \gamma\right)=\psi\left(\tau, z_{T}+X \beta, X ; \gamma+\beta\right)$. Therefore, we have

$$
\psi\left[\tau, \frac{z_{T}}{\sigma_{2, t}}, X A ; \gamma_{s t}\right]=\frac{1}{\sigma_{2, t}} \psi\left(\tau, z_{T}, X ; \gamma\right)
$$

and take the first order derivatives

$$
\frac{\partial}{\partial \gamma} \psi\left(\tau, \frac{z_{T}}{\sigma_{2, t}}, X A ; \gamma_{s t}\right)=\frac{1}{\sigma_{2, t}} \frac{\partial}{\partial \gamma} \psi\left(\tau, z_{T}, X ; \gamma\right)
$$


where

$$
A=\left(\begin{array}{cccc}
1 & 0 & 0 & 0 \\
0 & 1 & 0 & 0 \\
0 & 0 & \frac{1}{\sigma_{2, t}} & 0 \\
0 & 0 & 0 & \frac{1}{\sigma_{2, t}}
\end{array}\right)
$$

and $\gamma_{s t}=A^{-1} \gamma$ denotes the coefficient after the scaling transformation.

Note that the first order derivative of $\psi$ is equivalent to the score of the log likelihood function of the asymmetric Laplace density if we fix $\tau$ and $\sigma$. Thus (7.1) implies $s_{s t}(\gamma)=$ $\frac{1}{\sigma_{2, t}} s(\gamma)$, where $s_{s t}(\gamma)$ is the score function in the scaling transformation model. Similarly, we have the invariant property of the information matrix and other matrices,

$$
J_{s t}=\left(\frac{1}{\sigma_{2, t}}\right)^{2} J \quad \text { and } \quad K_{s t}=\frac{1}{\sigma_{2, t}} K ;
$$

therefore $V_{s t}=V$ where the subscript "st" denotes the corresponding variables after the scaling transformation.

Hence, we have

$$
\begin{aligned}
R S_{s t} & =\frac{1}{T} \tilde{s}_{2, s t}^{\prime} \tilde{K}_{s t}^{B B} \tilde{V}_{B B, s t} \tilde{K}_{s t}^{B B} \tilde{s}_{2, s t} \\
& =\frac{1}{T}\left(\frac{1}{\sigma_{2, t}} \tilde{s}_{2}^{\prime}\right)\left(\sigma_{2, t} \tilde{K}^{B B}\right) \tilde{V}_{B B}\left(\sigma_{2, t} \tilde{K}^{B B}\right)\left(\frac{1}{\sigma_{2, t}} \tilde{s}_{2}\right) \\
& =R S,
\end{aligned}
$$

which shows that Rao's score statistic in the regression quantile is invariant to the scaling transformation. The invariance property of the scaling transformation suggests a direct test procedure for contagion simply by usage of the unscaled parameters instead of the scaled ones. 


\section{Appendix 2: Derivation of Parameterization in the Monte Carlo Simula- tion}

In this appendix, the derivation of the parameterization in the Monte Carlo simulation is provided.

Suppose the non-crisis model is the combination of the common factor $w_{t}$ and the idiosyncratic factor $u_{i, t}$. Then

$$
x_{i, t}=\lambda_{i} w_{t}+\phi_{i} u_{i, t}
$$

where $\lambda_{i}$ and $\phi_{i}$ denote the loadings of the two factors. The crisis period model is

$$
y_{1, t}=\lambda_{1} w_{t}+\phi_{1} u_{1, t}, \quad \text { and } \quad y_{2, t}=\lambda_{2} w_{t}+\phi_{2} u_{2, t}+\delta \phi_{1} u_{1, t}
$$

where $\delta$ captures the strength of the contagion effect. For convenience, we define $v_{i, t}=$ $\lambda_{i} w_{t}+\phi_{i} u_{i, t}$ and $c_{1, t}=\phi_{1} u_{1, t}$ then the crisis model for country $i$ can be rewritten as

$$
y_{1, t}=v_{1, t} \quad \text { and } \quad y_{2, t}=v_{2, t}+\delta c_{1, t},
$$

In this setup, we show that the correlation between the two asset returns in the crisis period $y_{1}$ and $y_{2}$ is not necessarily monotonic with respect to $\delta$ as follows.

Consider the denominator and numerator in the correlation

$$
\operatorname{corr}\left(y_{1}, y_{2}\right)=\frac{\operatorname{Cov}\left(y_{1}, y_{2}\right)}{\sqrt{\operatorname{Var}\left(y_{1}\right) \operatorname{Var}\left(y_{2}\right)}} .
$$

Expanding the numerator and denominator we can explicitly express the correlation as a function of $\delta$

$$
\begin{aligned}
\operatorname{corr}\left(y_{1}, y_{2}\right) & =\frac{E\left(v_{1} v_{2}\right)-E\left(v_{1}\right) E\left(v_{2}\right)+\delta\left[E\left(v_{1} c_{1}\right)-E\left(v_{1}\right) E\left(c_{1}\right)\right]}{\sqrt{\operatorname{Var}\left(v_{1}\right) \operatorname{Var}\left(v_{2}\right)+\delta^{2} \operatorname{Var}\left(v_{1}\right) \operatorname{Var}\left(c_{1}\right)+2 \delta \operatorname{Var}\left(v_{1}\right) \operatorname{Cov}\left(v_{2}, c_{1}\right)}} \\
& =\frac{D E\left(v_{1}, v_{2}\right)+\delta D E\left(v_{1}, c_{1}\right)}{\sqrt{P V}},
\end{aligned}
$$


where $D E\left(v_{1}, c_{1}\right)=E\left(v_{1} c_{1}\right)-E\left(v_{1}\right) E\left(c_{1}\right), D E\left(v_{1}, v_{2}\right)=E\left(v_{1} v_{2}\right)-E\left(v_{1}\right) E\left(v_{2}\right)$ and $P V=\operatorname{Var}\left(v_{1}\right) \operatorname{Var}\left(v_{2}\right)+$ $\delta^{2} \operatorname{Var}\left(v_{1}\right) \operatorname{Var}\left(c_{1}\right)+2 \delta \operatorname{Var}\left(v_{1}\right) \operatorname{Cov}\left(v_{2}, c_{1}\right)$.

To study the behavior of the correlation with the increasing contagion effect $\delta$, we take the first order derivatives of Eq.(7.4) with respect to $\delta$

$$
\begin{gathered}
\frac{\partial \operatorname{corr}\left(y_{1}, y_{2}\right)}{\partial \delta}=\frac{\frac{\partial \operatorname{Cov}\left(y_{1}, y_{2}\right)}{\partial \delta} \sqrt{P V}-\operatorname{Cov}\left(y_{1}, y_{2}\right) \frac{\partial \sqrt{P V}}{\partial \delta}}{\sqrt{P V}} \\
=\frac{D E\left(v_{1}, c_{1}\right)-\frac{1}{2}\left[D E\left(v_{1}, v_{2}\right)+\delta D E\left(v_{1}, c_{1}\right)\right]\left[2 \delta \operatorname{Var}\left(v_{1}\right) \operatorname{Var}\left(c_{1}\right)+2 \operatorname{Var}\left(v_{1}\right) \operatorname{Cov}\left(v_{2}, c_{1}\right)\right] / P V}{P V^{3 / 2}}
\end{gathered}
$$

In order to determine the sign of the first order derivatives, we focus on the sign of the numerator since the denominator is guaranteed to be positive. The numerator can be rewritten as

$$
\frac{D E\left(v_{1}, c_{1}\right) P V-\frac{1}{2}\left[D E\left(v_{1}, v_{2}\right)+\delta D E\left(v_{1}, c_{1}\right)\right]\left[2 \delta \operatorname{Var}\left(v_{1}\right) \operatorname{Var}\left(c_{1}\right)+2 \operatorname{Var}\left(v_{1}\right) \operatorname{Cov}\left(v_{2}, c_{1}\right)\right]}{P V}
$$

and its sign is determined by its numerator

$$
\begin{aligned}
\text { Num }= & D E\left(v_{1}, c_{1}\right) \operatorname{Var}\left(v_{1}\right) \operatorname{Var}\left(v_{2}\right)-D E\left(v_{1}, v_{2}\right) \operatorname{Var}\left(v_{1}\right) \operatorname{Cov}\left(v_{2}, c_{1}\right) \\
& +\delta\left[D E\left(v_{1}, c_{1}\right) \operatorname{Var}\left(v_{1}\right) \operatorname{Cov}\left(v_{2}, c_{1}\right)-D E\left(v_{1}, v_{2}\right) \operatorname{Var}\left(v_{1}\right) \operatorname{Var}\left(c_{1}\right)\right]
\end{aligned}
$$

Note that the sign of Num depends on the parameterization. In this sense, we study its sign by assuming the distribution of random variables.

We consider that the data generation process is based on the normal distribution ${ }^{6}$. Suppose that $w_{t}, u_{1, t}$ and $u_{2, t}$ are independently distributed as

$$
w_{t} \sim N\left(\mu_{w}, \sigma_{w}^{2}\right), \quad u_{1, t} \sim N\left(\mu_{u_{1}}, \sigma_{u_{1}}^{2}\right), \quad u_{2, t} \sim N\left(\mu_{u_{2}}, \sigma_{u_{2}}^{2}\right)
$$

then we can obtain the distribution of $v_{1, t}, v_{2, t}$ and $c_{1, t}$ from the linearity of the normal distribution as

$$
v_{1, t} \sim N\left(\lambda_{1} \mu_{w}+\phi_{1} \mu_{u_{1}}, \lambda_{1}^{2} \sigma_{w}^{2}+\phi_{1}^{2} \sigma_{u_{1}}^{2}\right), \quad v_{2, t} \sim N\left(\lambda_{2} \mu_{w}+\phi_{2} \mu_{u_{2}}, \lambda_{2}^{2} \sigma_{w}^{2}+\phi_{2}^{2} \sigma_{u_{2}}^{2}\right),
$$

\footnotetext{
${ }^{6}$ The cases of the $t$ distribution and skewed $t$ distribution follow similar patterns to the normal case.
} 


$$
c_{1, t} \sim N\left(\phi_{1} \mu_{u_{1}}, \phi_{1}^{2} \sigma_{u_{1}}^{2}\right) .
$$

Furthermore, we express each term in Num with the parameter definition above as follows

$$
\begin{aligned}
& D E\left(v_{1}, c_{1}\right)=E\left(v_{1} c_{1}\right)-E\left(v_{1}\right) E\left(c_{1}\right)=\phi_{1}^{2} \sigma_{u_{1}}^{2}, \\
& D E\left(v_{1}, v_{2}\right)=E\left(v_{1} v_{2}\right)-E\left(v_{1}\right) E\left(v_{2}\right)=\lambda_{1} \lambda_{2} \sigma_{w}^{2}, \\
& \operatorname{Cov}\left(v_{2}, c_{1}\right)=0 .
\end{aligned}
$$

The last equation holds because $v_{2}$ and $c_{1}$ are assumed to be independent in this model. Therefore, Num can be written as

$$
N u m=\phi_{1}^{2} \sigma_{u_{1}}^{2}\left(\lambda_{1}^{2} \sigma_{w}^{2}+\phi_{1}^{2} \sigma_{u_{1}}^{2}\right)\left(\lambda_{2}^{2} \sigma_{w}^{2}+\phi_{2}^{2} \sigma_{u_{2}}^{2}-\delta \lambda_{1} \lambda_{2} \sigma_{w}^{2}\right) .
$$

It can be verified that when

$$
\delta>\frac{\lambda_{2}^{2} \sigma_{w}^{2}+\phi_{2}^{2} \sigma_{u_{2}}^{2}}{\lambda_{1} \lambda_{2} \sigma_{w}^{2}}
$$

increasing $\delta$ can lead to a negative and decreasing Num and, therefore, a decreasing correlation. 

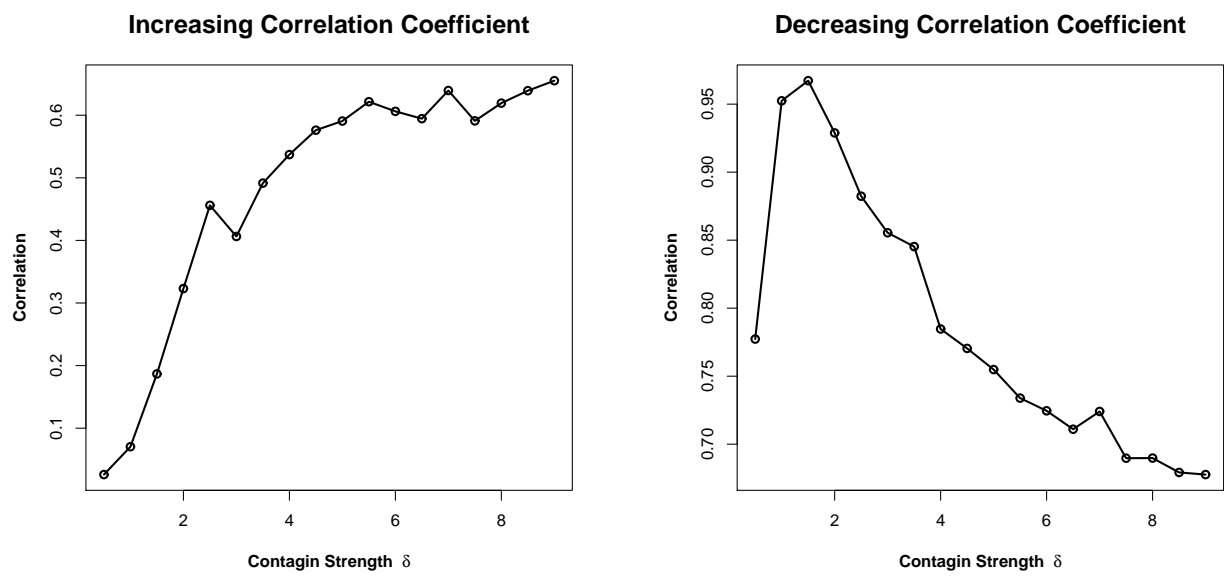

Figure 1: Correlation under Different Parameterizations 

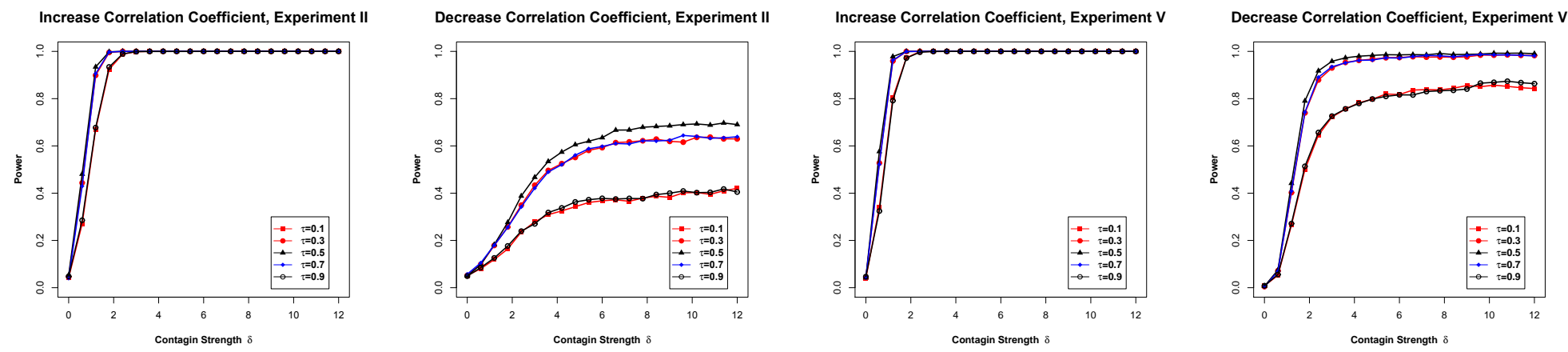

吕
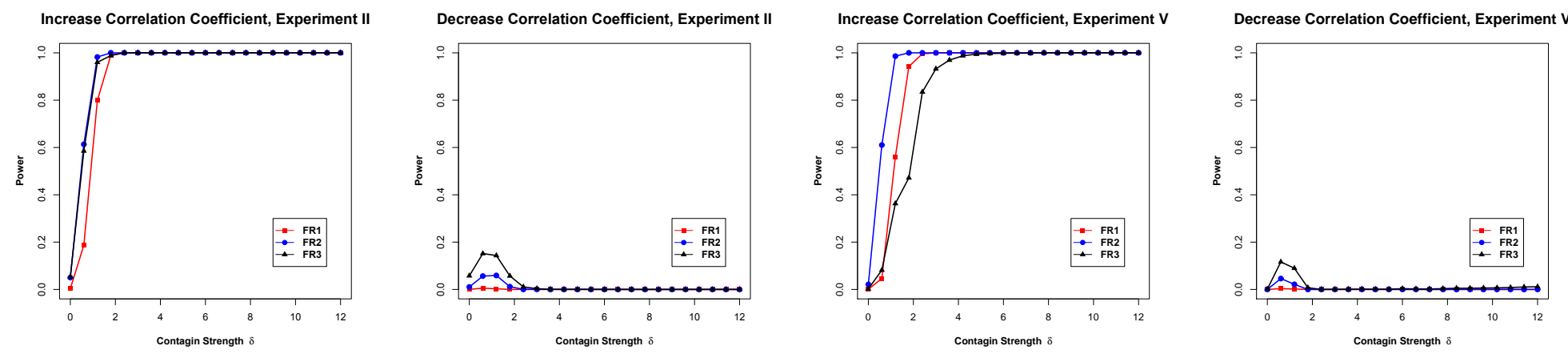

Figure 2: Power Property under the Normal Distribution with Sample Size 200 

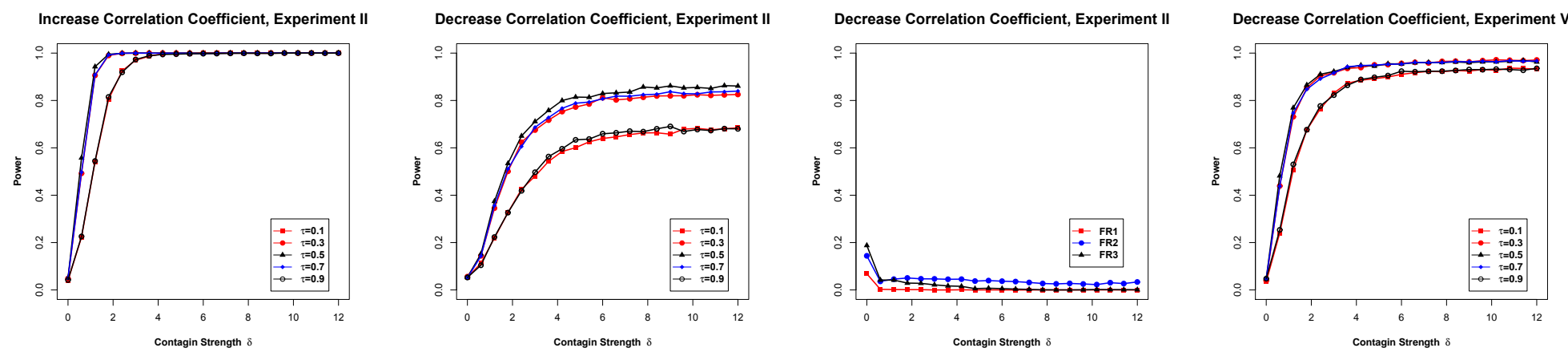

斗
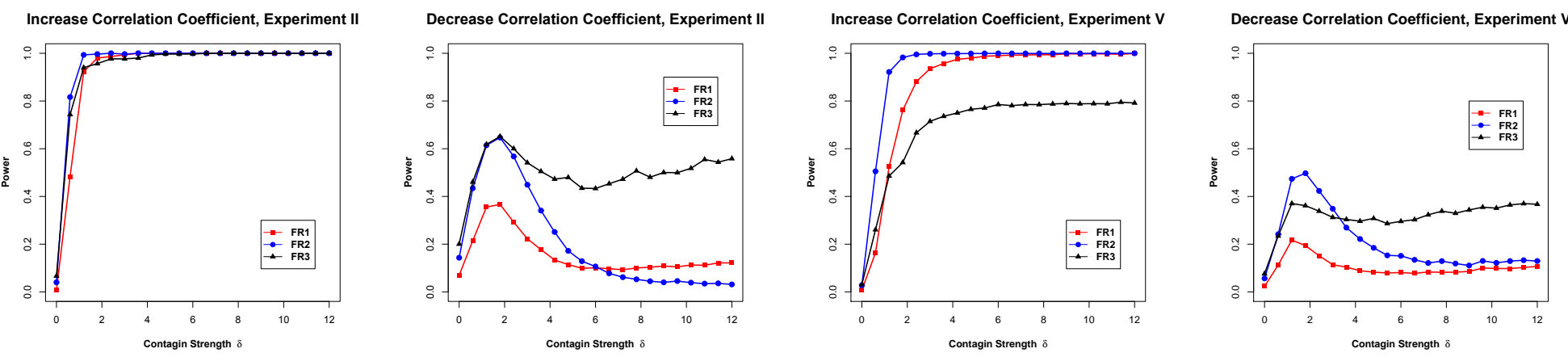

Figure 3: Power Property under the Student's $t$ Distribution with Sample Size 200 

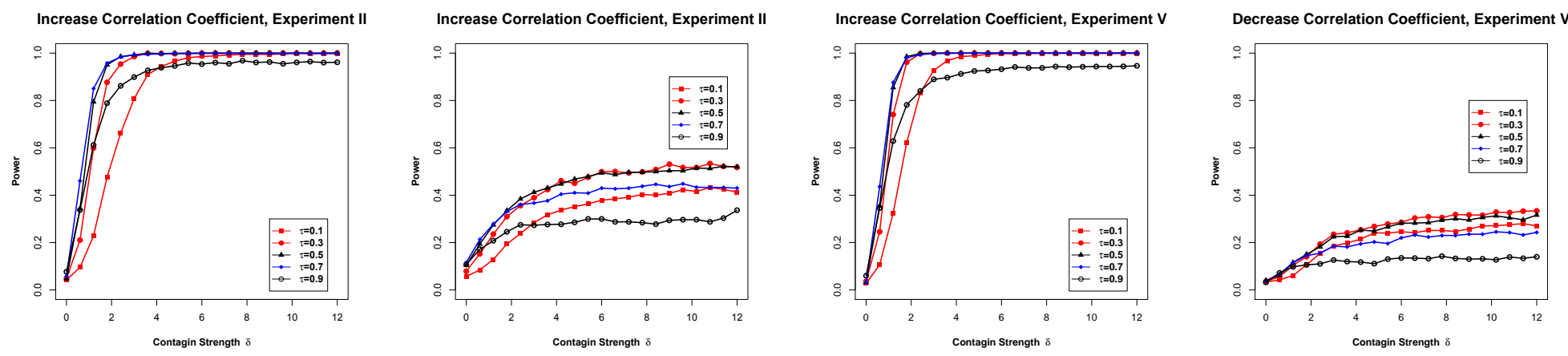

G
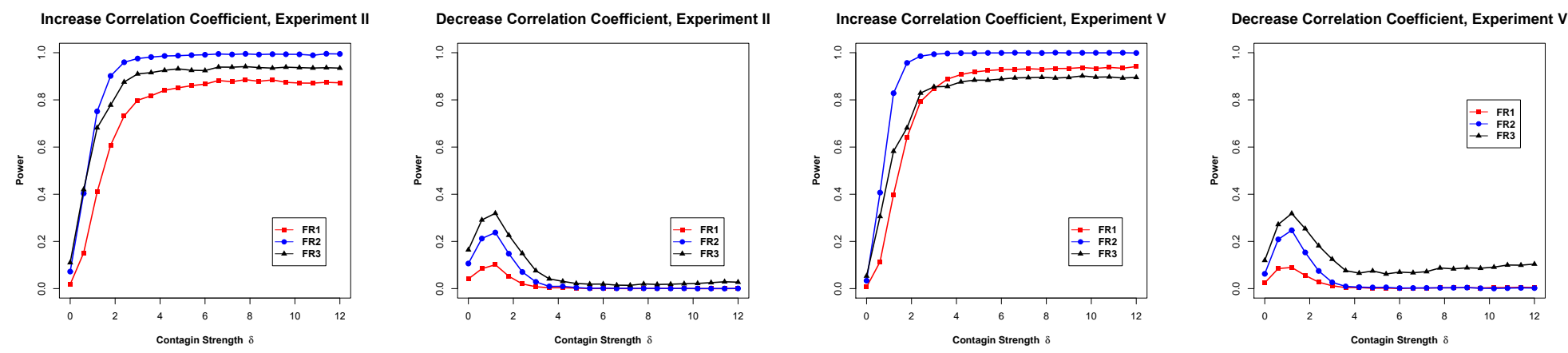

Figure 4: Power Property under the Skewed $t$ Distribution with Sample Size 200 

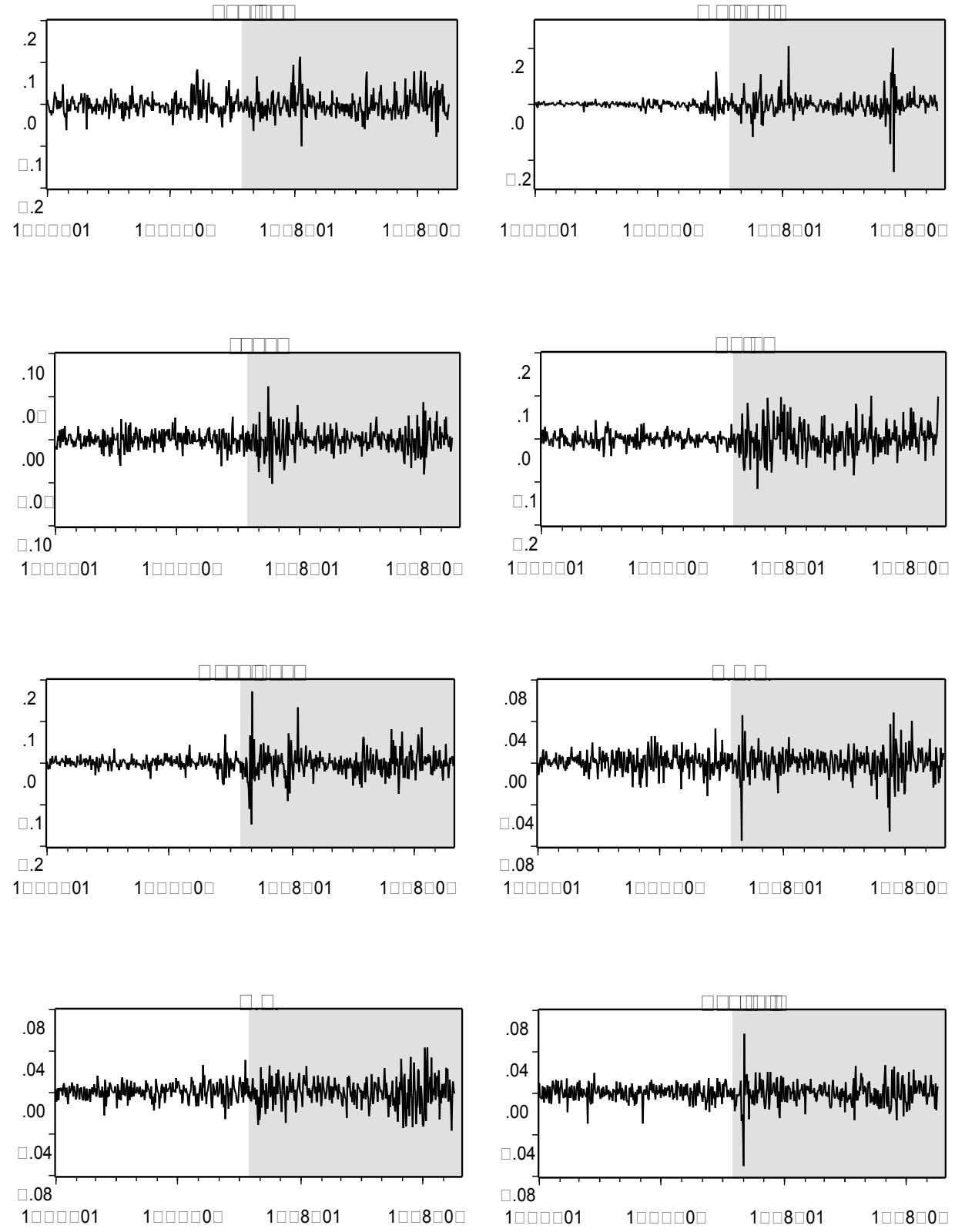

Figure 5: Daily percentage equity returns for some countries around the Asian Crisis. The start of the sample period is Jan 1, 1997 (vertical axis). The end of the sample period is Aug 31, 1998. The crisis period is from Oct 20, 1997 to Aug 31, 1998 (shaded area). 

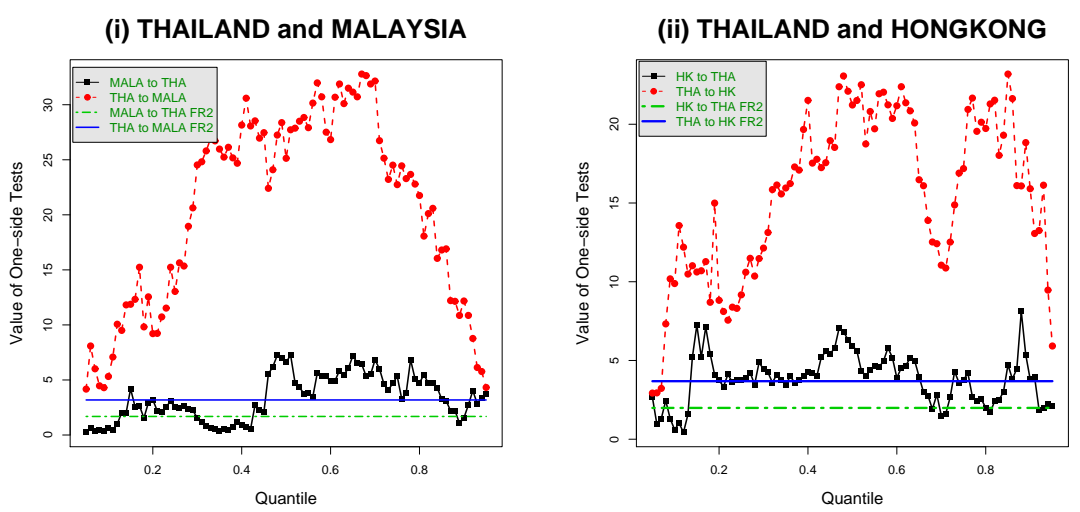

(iii) THAILAND and KOREA

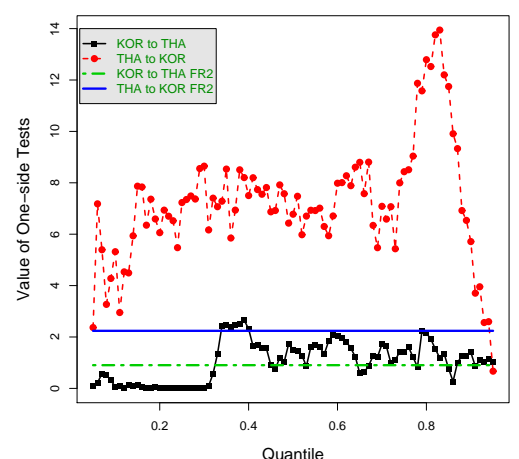

$\mathbb{v}$
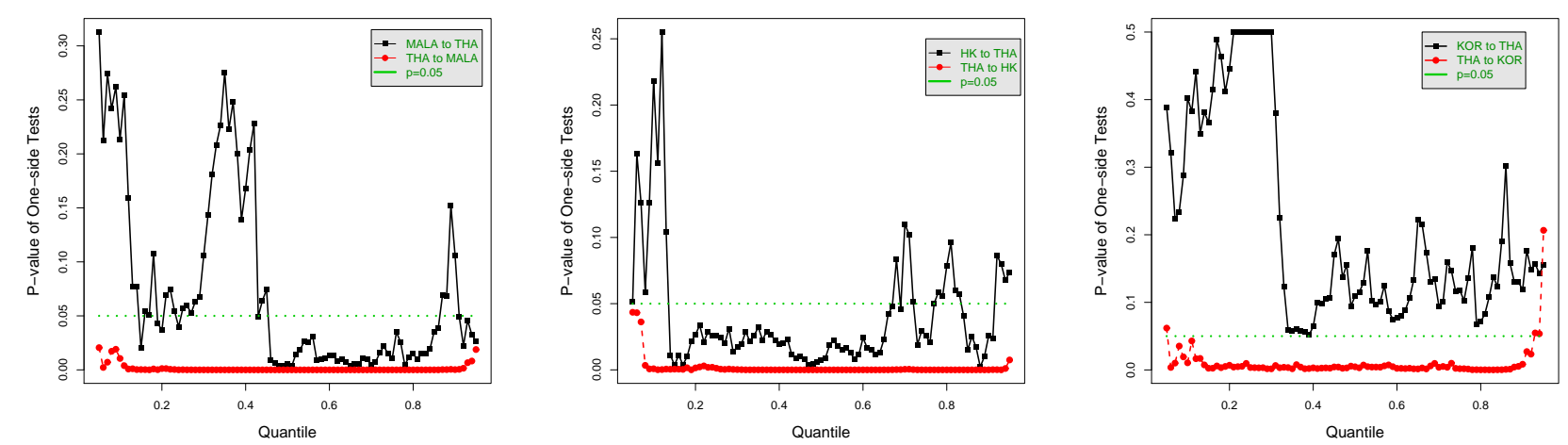

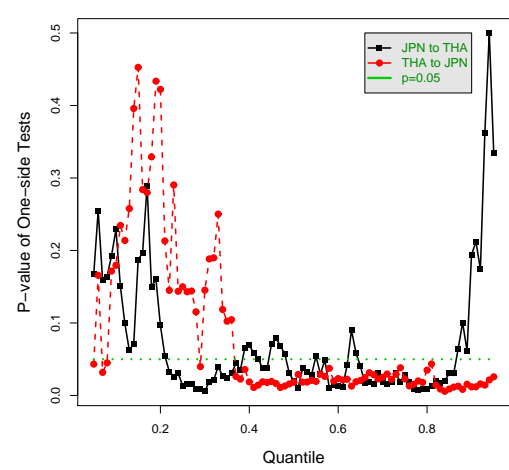

Figure 6: Statistics and $p$-value in the Asian Crisis: Within Asia 
(iii) JAPAN and U.S.

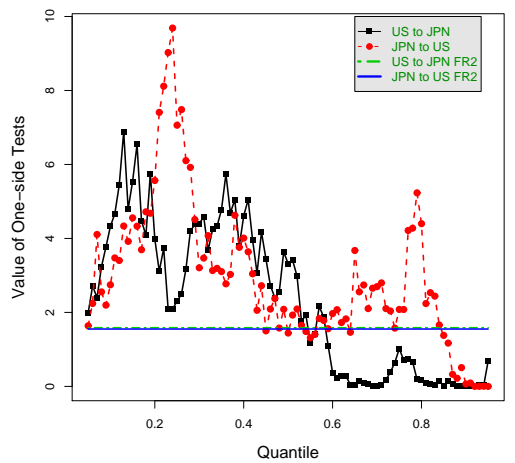

$\stackrel{\infty}{\infty}$

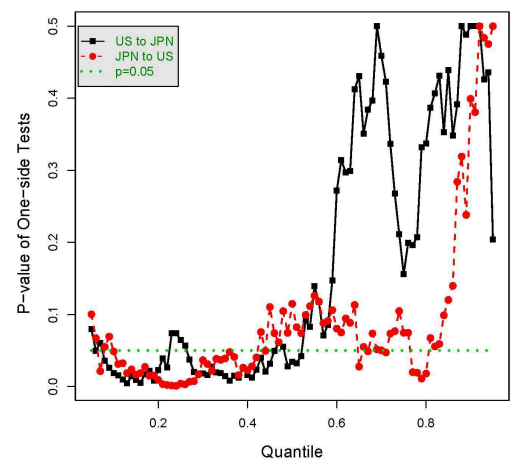

(iv) JAPAN and AUSTRALIA
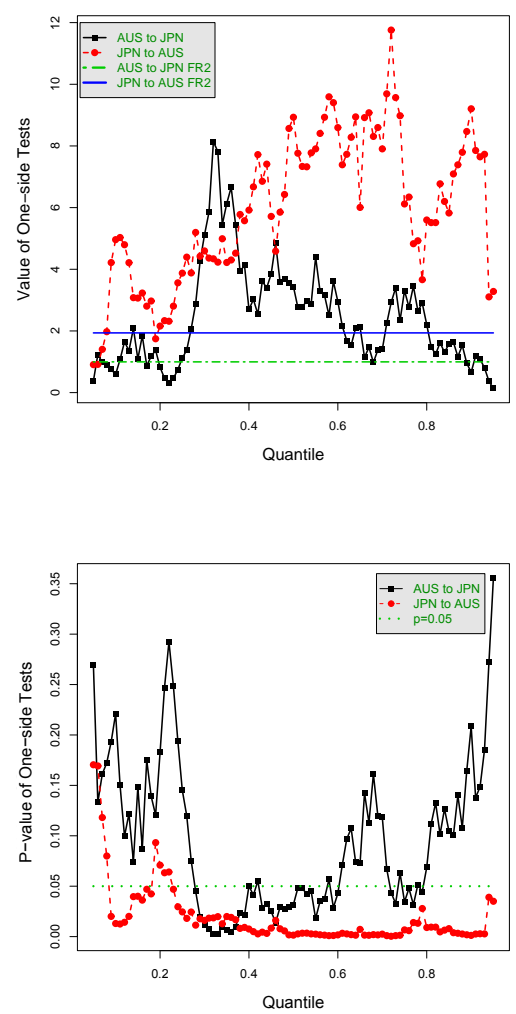

(i) HONGKONG and U.S.
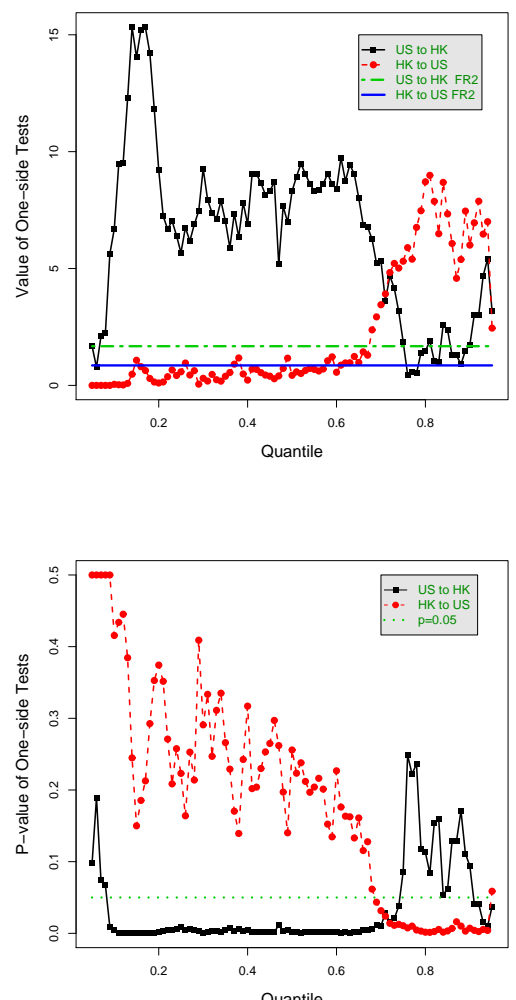

(ii) HONGKONG and U.K.
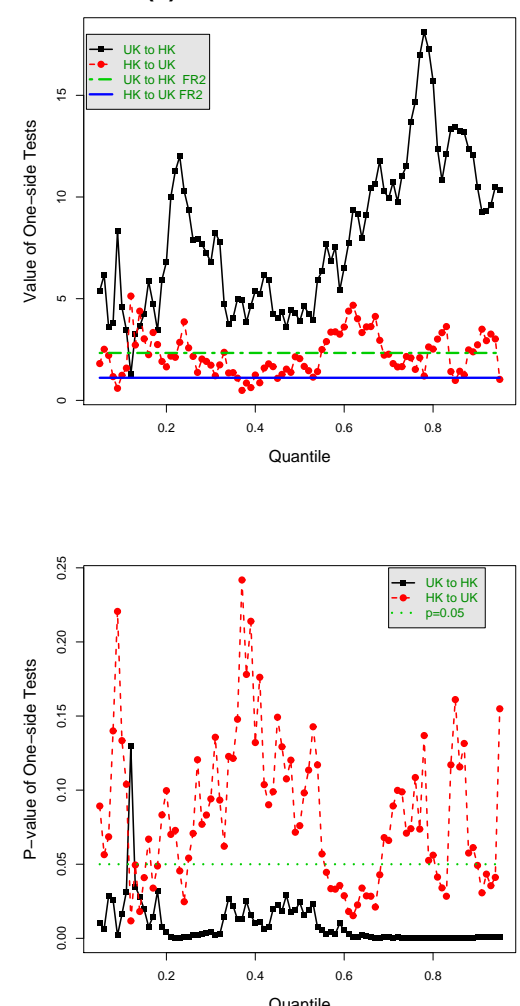

Figure 7: Statistics and $p$-value in the Asian Crisis: From Asia to the West 

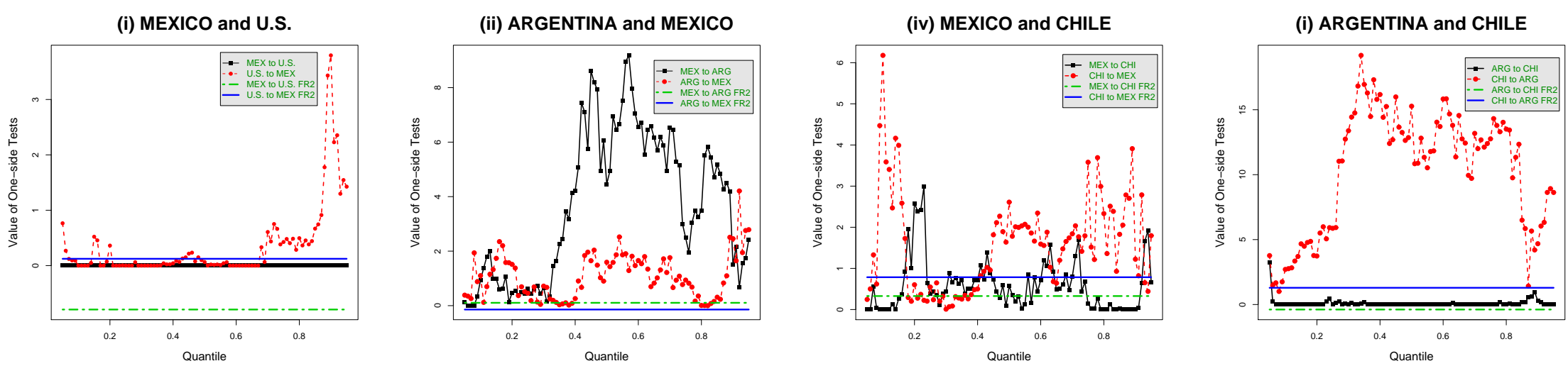

to
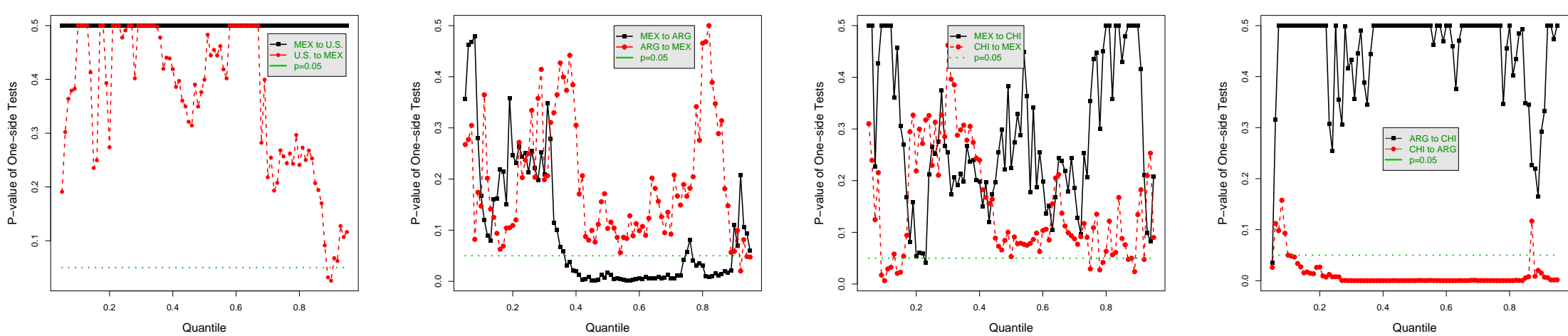

Figure 8: Statistics and $p$-value in the Tequila Crisis 

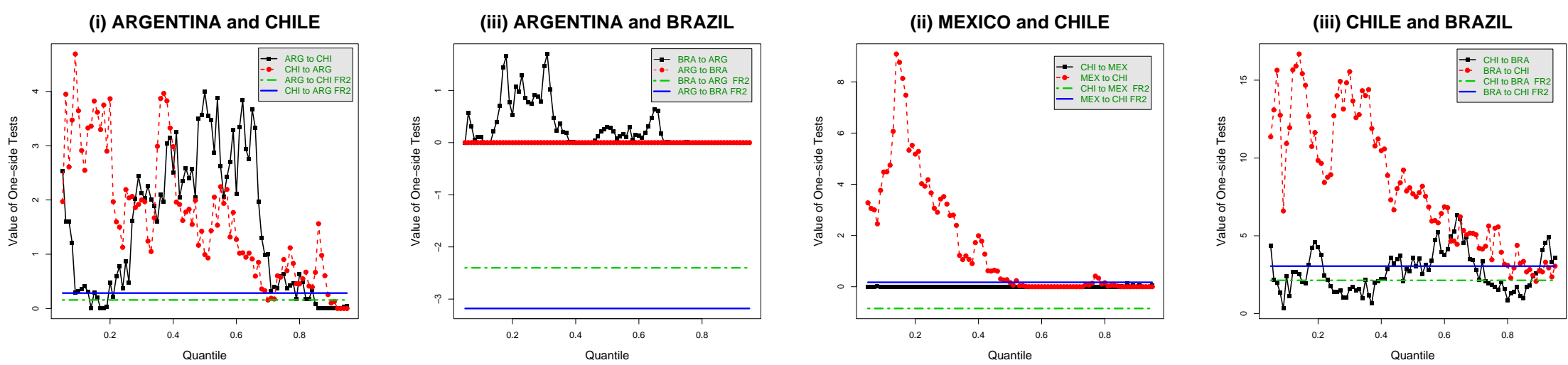

잉
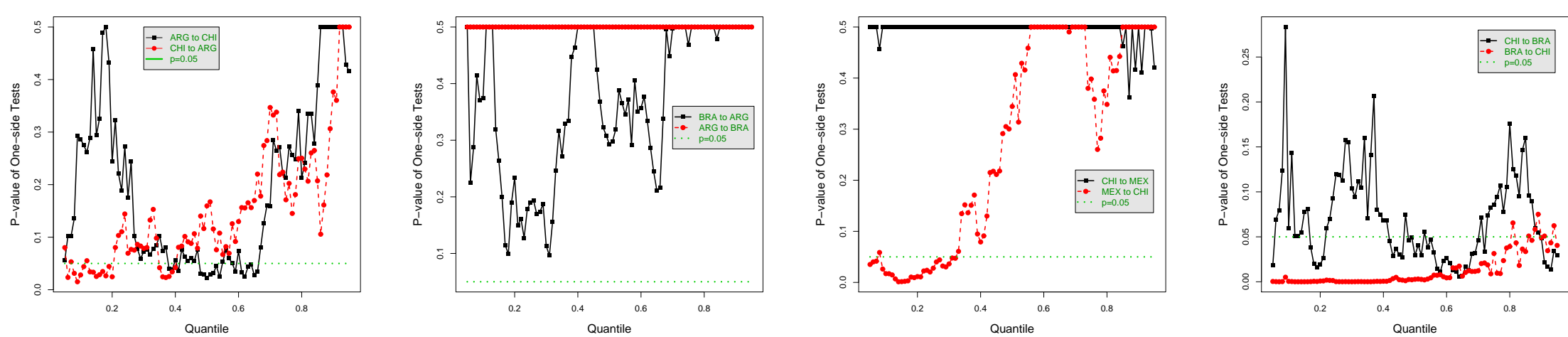

Figure 9: Statistics and $p$-value in the Argentine Crisis 
Table 1: Parameterization in the Experiments

\begin{tabular}{|c|c|c|c|c|c|c|c|}
\hline Normal Distribution & $\alpha$ & $\beta$ & $\rho$ & $\omega_{x}$ & $\omega_{y}$ & $\kappa_{x}$ & $\kappa_{y}$ \\
\hline \multicolumn{8}{|l|}{ Increasing Correlation } \\
\hline (I) Strong Autocorrelation & 0 & 0 & 0.95 & 0.50 & 0.50 & 4 & 4 \\
\hline (II) Weak Autocorrelation & 0 & 0 & 0.20 & 0.50 & 0.50 & 4 & 4 \\
\hline (III) No Autocorrelation & 0 & 0 & 0 & 0.50 & 0.50 & 4 & 4 \\
\hline (IV) Common Factor Structural Break & 0 & 0 & 0 & 0.50 & 1 & 4 & 4 \\
\hline (V) Idiosyncratic Factor Structural & 0 & 0 & 0 & 0.50 & 0.50 & 4 & 6 \\
\hline (VI) GARCH & 0.05 & 0.95 & 0 & 0.50 & 0.50 & 4 & 4 \\
\hline \multicolumn{8}{|l|}{ Decreasing Correlation } \\
\hline (I) Strong Autocorrelation & 0 & 0 & 0.95 & 2 & 2 & 0.50 & 0.50 \\
\hline (II) Weak Autocorrelation & 0 & 0 & 0.20 & 2 & 2 & 0.50 & 0.50 \\
\hline (III) No Autocorrelation & 0 & 0 & 0 & 2 & 2 & 0.50 & 0.50 \\
\hline (IV) Common Factor Structural Break & 0 & 0 & 0 & 2 & 4 & 0.50 & 0.50 \\
\hline (V) Idiosyncratic Factor Structural & 0 & 0 & 0 & 2 & 2 & 0.50 & 1 \\
\hline (VI) GARCH & 0.10 & 0.90 & 0 & 4 & 4 & 0.01 & 0.01 \\
\hline Student's $t$ Distribution & $\alpha$ & $\beta$ & $\rho$ & $\omega_{x}$ & $\omega_{y}$ & $d f_{x}$ & $d f_{y}$ \\
\hline \multicolumn{8}{|l|}{ Increasing Correlation } \\
\hline (I) Strong Autocorrelation & 0 & 0 & 0.95 & 0.50 & 0.50 & 3 & 3 \\
\hline (II) Weak Autocorrelation & 0 & 0 & 0.20 & 0.50 & 0.50 & 3 & 3 \\
\hline (III) No Autocorrelation & 0 & 0 & 0 & 0.50 & 0.50 & 3 & 3 \\
\hline (IV) Common Factor Structural Break & 0 & 0 & 0 & 0.50 & 1 & 3 & 3 \\
\hline (V) Idiosyncratic Factor Structural & 0 & 0 & 0 & 0.50 & 0.50 & 3 & 2 \\
\hline (VI) GARCH & 0.05 & 0.95 & 0 & 0.50 & 0.50 & 3 & 3 \\
\hline \multicolumn{8}{|l|}{ Decreasing Correlation } \\
\hline (I) Strong Autocorrelation & 0 & 0 & 0.95 & 2 & 2 & 3 & 3 \\
\hline (II) Weak Autocorrelation & 0 & 0 & 0.20 & 2 & 2 & 3 & 3 \\
\hline (III) No Autocorrelation & 0 & 0 & 0 & 2 & 2 & 3 & 3 \\
\hline (IV) Common Factor Structural Break & 0 & 0 & 0 & 2 & 4 & 3 & 3 \\
\hline (V) Idiosyncratic Factor Structural & 0 & 0 & 0 & 2 & 2 & 3 & 2 \\
\hline (VI) GARCH & 0.10 & 0.90 & 0 & 4 & 4 & 3 & 3 \\
\hline Skew $t$ Distribution & $\alpha$ & $\beta$ & $\rho$ & $\omega_{x}$ & $\omega_{y}$ & $\phi_{x}$ & $\phi_{y}$ \\
\hline \multicolumn{8}{|l|}{ Increasing Correlation } \\
\hline (I) Strong Autocorrelation & 0 & 0 & 0.95 & 0.50 & 0.50 & -0.3 & -0.3 \\
\hline (II) Weak Autocorrelation & 0 & 0 & 0.20 & 0.50 & 0.50 & -0.3 & -0.3 \\
\hline (III) No Autocorrelation & 0 & 0 & 0 & 0.50 & 0.50 & -0.3 & -0.3 \\
\hline (IV) Common Factor Structural Break & 0 & 0 & 0 & 0.15 & 0.30 & -0.3 & -0.3 \\
\hline (V) Idiosyncratic Factor Structural & 0 & 0 & 0 & 0.50 & 0.50 & -0.3 & -0.7 \\
\hline (VI) GARCH & 0.05 & 0.95 & 0 & 0.50 & 0.50 & -0.3 & -0.3 \\
\hline \multicolumn{8}{|l|}{ Decreasing Correlation } \\
\hline (I) Strong Autocorrelation & 0 & 0 & 0.95 & 2 & 2 & -0.3 & -0.3 \\
\hline (II) Weak Autocorrelation & 0 & 0 & 0.20 & 2 & 2 & -0.3 & -0.3 \\
\hline (III) No Autocorrelation & $0 \quad 51$ & 0 & 0 & 2 & 2 & -0.3 & -0.3 \\
\hline (IV) Common Factor Structural Break & 0 & 0 & 0 & 2 & 4 & -0.3 & -0.3 \\
\hline (V) Idiosyncratic Factor Structural & 0 & 0 & 0 & 2 & 2 & -0.3 & -0.7 \\
\hline (VI) GARCH & 0.10 & 0.90 & 0 & 4 & 4 & -0.3 & -0.3 \\
\hline
\end{tabular}

Notes: The parameter $v$ in (6.5) equals 4 for all experiment designs in the skewed $t$ distribution. 
Table 2: Size Property under Increasing Correlation Cases

\begin{tabular}{|c|c|c|c|c|c|c|c|c|c|c|c|c|c|c|c|c|c|c|c|}
\hline \multirow{2}{*}{$\begin{array}{c}\text { Normal } \\
\text { Distribution }\end{array}$} & & \multicolumn{6}{|c|}{ sample size 200} & \multicolumn{6}{|c|}{ sample size 500} & \multicolumn{6}{|c|}{ sample size 1000} \\
\hline & & I & II & III & IV & $\mathrm{V}$ & VI & I & II & III & IV & $\mathrm{V}$ & VI & I & II & III & IV & $\mathrm{V}$ & VI \\
\hline \multirow[t]{2}{*}{$\tau=0.10$} & $Q S T$ & 0.0507 & 0.0487 & 0.0500 & 0.0530 & 0.0650 & 0.0563 & 0.0453 & 0.0460 & 0.0487 & 0.0577 & 0.0533 & 0.0513 & 0.0560 & 0.0497 & 0.0537 & 0.0660 & 0.0617 & 0.0483 \\
\hline & $Q O T$ & 0.0393 & 0.0497 & 0.0467 & 0.0613 & 0.0383 & 0.0407 & 0.0327 & 0.0440 & 0.0467 & 0.0987 & 0.0350 & 0.0443 & 0.0283 & 0.0477 & 0.0447 & 0.1270 & 0.0380 & 0.0450 \\
\hline \multirow[t]{2}{*}{$\tau=0.30$} & $Q S T$ & 0.0610 & 0.0510 & 0.0550 & 0.0653 & 0.0647 & 0.0573 & 0.0547 & 0.0513 & 0.0470 & 0.0717 & 0.0600 & 0.0547 & 0.0530 & 0.0513 & 0.0523 & 0.0807 & 0.0543 & 0.0447 \\
\hline & $Q O T$ & 0.0387 & 0.0510 & 0.0510 & 0.0970 & 0.0510 & 0.0520 & 0.0273 & 0.0487 & 0.0410 & 0.1253 & 0.0377 & 0.0490 & 0.0267 & 0.0513 & 0.0507 & 0.1763 & 0.0320 & 0.0467 \\
\hline \multirow{2}{*}{$\tau=0.50$} & $Q S T$ & 0.0547 & 0.0493 & 0.0513 & 0.0597 & 0.0567 & 0.0520 & 0.0657 & 0.0460 & 0.0390 & 0.0700 & 0.0520 & 0.0530 & 0.0640 & 0.0593 & 0.0557 & 0.0837 & 0.0527 & 0.0570 \\
\hline & $Q O T$ & 0.0347 & 0.0427 & 0.0493 & 0.0930 & 0.0460 & 0.0517 & 0.0277 & 0.0487 & 0.0460 & 0.1237 & 0.0313 & 0.0513 & 0.0213 & 0.0530 & 0.0500 & 0.1677 & 0.0377 & 0.0543 \\
\hline \multirow[t]{2}{*}{$\tau=0.70$} & $Q S T$ & 0.0517 & 0.0557 & 0.0413 & 0.0640 & 0.0587 & 0.0523 & 0.0573 & 0.0457 & 0.0470 & 0.0623 & 0.0520 & 0.0467 & 0.0640 & 0.0470 & 0.0557 & 0.0857 & 0.0477 & 0.0480 \\
\hline & $Q O T$ & 0.0343 & 0.0523 & 0.0517 & 0.0933 & 0.0483 & 0.0547 & 0.0297 & 0.0453 & 0.0490 & 0.1120 & 0.0357 & 0.0477 & 0.0217 & 0.0450 & 0.0530 & 0.1743 & 0.0410 & 0.0507 \\
\hline \multirow[t]{5}{*}{$\tau=0.90$} & $Q S T$ & 0.0487 & 0.0593 & 0.0563 & 0.0633 & 0.0617 & 0.0553 & 0.0493 & 0.0480 & 0.0477 & 0.0617 & 0.0560 & 0.0560 & 0.0617 & 0.0493 & 0.0507 & 0.0733 & 0.0513 & 0.0503 \\
\hline & $Q O T$ & 0.0367 & 0.0587 & 0.0527 & 0.0750 & 0.0423 & 0.0547 & 0.0307 & 0.0443 & 0.0543 & 0.0913 & 0.0410 & 0.0527 & 0.0287 & 0.0483 & 0.0480 & 0.1373 & 0.0337 & 0.0510 \\
\hline & $F R_{1}$ & 0.0027 & 0.0040 & 0.0030 & 0.0043 & 0.0000 & 0.0030 & 0.0013 & 0.0047 & 0.0033 & 0.0087 & 0.0000 & 0.0010 & 0.0000 & 0.0020 & 0.0017 & 0.0140 & 0.0000 & 0.0023 \\
\hline & $F R_{2}$ & 0.0343 & 0.0470 & 0.0470 & 0.0890 & 0.0197 & 0.0520 & 0.0310 & 0.0517 & 0.0487 & 0.1333 & 0.0223 & 0.0553 & 0.0243 & 0.0433 & 0.0493 & 0.2007 & 0.0173 & 0.0480 \\
\hline & $F R_{3}$ & 0.0470 & 0.0500 & 0.0490 & 0.0673 & 0.0017 & 0.0547 & 0.0423 & 0.0520 & 0.0477 & 0.0980 & 0.0017 & 0.0567 & 0.0433 & 0.0460 & 0.0507 & 0.1443 & 0.0020 & 0.0513 \\
\hline Student's $t$ & & \multicolumn{6}{|c|}{ sample size 200} & \multicolumn{6}{|c|}{ sample size 500} & \multicolumn{6}{|c|}{ sample size 1000} \\
\hline Distribution & & $\mathrm{I}$ & II & III & IV & $\mathrm{V}$ & $\mathrm{VI}$ & $\mathrm{I}$ & II & III & IV & $\mathrm{V}$ & $\mathrm{VI}$ & $\mathrm{I}$ & II & III & IV & $\mathrm{V}$ & VI \\
\hline \multirow[t]{2}{*}{$\tau=0.10$} & $Q S T$ & 0.0677 & 0.0563 & 0.0677 & 0.1117 & 0.1140 & 0.0707 & 0.0720 & 0.0567 & 0.0640 & 0.1383 & 0.1217 & 0.0600 & 0.0830 & 0.0700 & 0.0577 & 0.2400 & 0.1383 & 0.0487 \\
\hline & QOT & 0.0340 & 0.0447 & 0.0393 & 0.1423 & 0.0277 & 0.0360 & 0.0293 & 0.0427 & 0.0510 & 0.2650 & 0.0180 & 0.0453 & 0.0203 & 0.0653 & 0.0537 & 0.4147 & 0.0143 & 0.0360 \\
\hline \multirow[t]{2}{*}{$\tau=0.30$} & $Q S T$ & 0.0667 & 0.0593 & 0.0670 & 0.1317 & 0.1090 & 0.0660 & 0.0690 & 0.0527 & 0.0587 & 0.2830 & 0.1163 & 0.0667 & 0.0880 & 0.0517 & 0.0603 & 0.5447 & 0.1350 & 0.0470 \\
\hline & $Q O T$ & 0.0323 & 0.0463 & 0.0597 & 0.2647 & 0.0223 & 0.0520 & 0.0153 & 0.0533 & 0.0540 & 0.4957 & 0.0120 & 0.0570 & 0.0073 & 0.0553 & 0.0587 & 0.7737 & 0.0060 & 0.0437 \\
\hline \multirow[t]{2}{*}{$\tau=0.50$} & $Q S T$ & 0.0587 & 0.0510 & 0.0570 & 0.1523 & 0.0983 & 0.0633 & 0.0653 & 0.0460 & 0.0490 & 0.3347 & 0.1083 & 0.0580 & 0.0993 & 0.0573 & 0.0523 & 0.6397 & 0.1400 & 0.0537 \\
\hline & $Q O T$ & 0.0270 & 0.0483 & 0.0483 & 0.2987 & 0.0207 & 0.0603 & 0.0123 & 0.0490 & 0.0567 & 0.5590 & 0.0097 & 0.0523 & 0.0037 & 0.0600 & 0.0537 & 0.8390 & 0.0033 & 0.0457 \\
\hline \multirow[t]{2}{*}{$\tau=0.70$} & $Q S T$ & 0.0603 & 0.0540 & 0.0657 & 0.1563 & 0.1047 & 0.0673 & 0.0723 & 0.0530 & 0.0623 & 0.2967 & 0.1117 & 0.0590 & 0.0890 & 0.0560 & 0.0497 & 0.5627 & 0.1403 & 0.0503 \\
\hline & $Q O T$ & 0.0243 & 0.0477 & 0.0497 & 0.2727 & 0.0247 & 0.0570 & 0.0140 & 0.0477 & 0.0570 & 0.4987 & 0.0127 & 0.0503 & 0.0087 & 0.0590 & 0.0497 & 0.7710 & 0.0037 & 0.0520 \\
\hline \multirow[t]{5}{*}{$\tau=0.90$} & $Q S T$ & 0.0737 & 0.0727 & 0.0817 & 0.1107 & 0.1540 & 0.0880 & 0.0773 & 0.0703 & 0.0637 & 0.1523 & 0.1517 & 0.0640 & 0.0800 & 0.0627 & 0.0690 & 0.2547 & 0.1710 & 0.0603 \\
\hline & $Q O T$ & 0.0253 & 0.0450 & 0.0497 & 0.1433 & 0.0330 & 0.0420 & 0.0290 & 0.0517 & 0.0527 & 0.2533 & 0.0247 & 0.0447 & 0.0210 & 0.0600 & 0.0597 & 0.4143 & 0.0130 & 0.0483 \\
\hline & $F R_{1}$ & 0.0193 & 0.0160 & 0.0163 & 0.0497 & 0.0060 & 0.0123 & 0.0287 & 0.0183 & 0.0187 & 0.0997 & 0.0053 & 0.0037 & 0.0280 & 0.0240 & 0.0223 & 0.3980 & 0.0007 & 0.0060 \\
\hline & $F R_{2}$ & 0.0697 & 0.0620 & 0.0737 & 0.2300 & 0.0293 & 0.0647 & 0.0907 & 0.0773 & 0.0767 & 0.4540 & 0.0150 & 0.0523 & 0.0900 & 0.0820 & 0.0820 & 0.8587 & 0.0070 & 0.0503 \\
\hline & $F R_{3}$ & 0.1040 & 0.0970 & 0.1060 & 0.1140 & 0.0347 & 0.0737 & 0.1330 & 0.1160 & 0.1143 & 0.1523 & 0.0150 & 0.0627 & 0.1290 & 0.1170 & 0.1170 & 0.4407 & 0.0063 & 0.0590 \\
\hline Skew $t$ & & \multicolumn{6}{|c|}{ sample size 200} & & & samp & ize 500 & & & & & sample & ze 1000 & & \\
\hline Distribution & & I & II & III & IV & $\mathrm{V}$ & $\mathrm{VI}$ & I & II & III & IV & $\mathrm{V}$ & $\mathrm{VI}$ & I & II & III & IV & $\mathrm{V}$ & $\mathrm{VI}$ \\
\hline$\tau=0.10$ & QST & 0.0457 & 0.0507 & 0.0400 & 0.0480 & 0.0467 & 0.0507 & 0.0407 & 0.0460 & 0.0457 & 0.0527 & 0.0443 & 0.0457 & 0.0450 & 0.0473 & 0.0410 & 0.0493 & 0.0493 & 0.0527 \\
\hline & QOT & 0.0237 & 0.0387 & 0.0330 & 0.0433 & 0.0287 & 0.0393 & 0.0243 & 0.0403 & 0.0427 & 0.0697 & 0.0297 & 0.0427 & 0.0197 & 0.0360 & 0.0420 & 0.0983 & 0.0263 & 0.0497 \\
\hline$\tau=0.30$ & QST & 0.0510 & 0.0477 & 0.0477 & 0.0583 & 0.0423 & 0.0537 & 0.0590 & 0.0510 & 0.0520 & 0.0803 & 0.0467 & 0.0520 & 0.0700 & 0.0467 & 0.0490 & 0.1023 & 0.0493 & 0.0517 \\
\hline & QOT & 0.0223 & 0.0497 & 0.0417 & 0.1017 & 0.0293 & 0.0473 & 0.0227 & 0.0527 & 0.0553 & 0.1717 & 0.0287 & 0.0553 & 0.0067 & 0.0410 & 0.0427 & 0.2390 & 0.0210 & 0.0537 \\
\hline$\tau=0.50$ & QST & 0.0577 & 0.0510 & 0.0533 & 0.0853 & 0.0597 & 0.0613 & 0.0690 & 0.0553 & 0.0607 & 0.1407 & 0.0660 & 0.0607 & 0.0953 & 0.0487 & 0.0473 & 0.2270 & 0.0667 & 0.0447 \\
\hline & $\mathrm{QOT}$ & 0.0237 & 0.0480 & 0.0563 & 0.1550 & 0.0300 & 0.0507 & 0.0173 & 0.0543 & 0.0570 & 0.2573 & 0.0257 & 0.0570 & 0.0090 & 0.0537 & 0.0480 & 0.4087 & 0.0163 & 0.0517 \\
\hline$\tau=0.70$ & QST & 0.0720 & 0.0663 & 0.0683 & 0.1123 & 0.0727 & 0.0597 & 0.0870 & 0.0650 & 0.0607 & 0.1893 & 0.0827 & 0.0607 & 0.1377 & 0.0590 & 0.0600 & 0.3387 & 0.1027 & 0.0517 \\
\hline & QOT & 0.0240 & 0.0677 & 0.0660 & 0.1937 & 0.0343 & 0.0493 & 0.0197 & 0.0630 & 0.0677 & 0.3433 & 0.0253 & 0.0677 & 0.0053 & 0.0603 & 0.0587 & 0.5397 & 0.0190 & 0.0437 \\
\hline$\tau=0.90$ & QST & 0.0973 & 0.0943 & 0.0883 & 0.1037 & 0.1130 & 0.0717 & 0.1180 & 0.0920 & 0.0957 & 0.1193 & 0.1237 & 0.0957 & 0.1697 & 0.0927 & 0.0807 & 0.1597 & 0.1383 & 0.0503 \\
\hline & $\mathrm{QOT}$ & 0.0383 & 0.0817 & 0.0747 & 0.1300 & 0.0487 & 0.0460 & 0.0367 & 0.1027 & 0.1017 & 0.2053 & 0.0420 & 0.1017 & 0.0147 & 0.0780 & 0.0833 & 0.2757 & 0.0290 & 0.0463 \\
\hline
\end{tabular}


Table 3: Size Property under Decreasing Correlation Cases

\begin{tabular}{|c|c|c|c|c|c|c|c|c|c|c|c|c|c|c|c|c|c|c|c|}
\hline & & \multicolumn{6}{|c|}{ sample size 200} & \multicolumn{6}{|c|}{ sample size 500} & \multicolumn{6}{|c|}{ sample size 1000} \\
\hline & & I & II & III & IV & $\mathrm{V}$ & VI & I & II & III & IV & $\mathrm{V}$ & VI & I & II & III & IV & $\mathrm{V}$ & VI \\
\hline \multirow[t]{2}{*}{$\tau=0.10$} & $Q S T$ & 0.0557 & 0.0510 & 0.0447 & 0.0700 & 0.0613 & 0.0543 & 0.0557 & 0.0520 & 0.0527 & 0.0700 & 0.1370 & 0.0460 & 0.0500 & 0.0453 & 0.0527 & 0.0610 & 0.0933 & 0.0483 \\
\hline & $Q O T$ & 0.0413 & 0.0437 & 0.0407 & 0.0533 & 0.0153 & 0.0413 & 0.0530 & 0.0493 & 0.0500 & 0.0533 & 0.0027 & 0.0450 & 0.0450 & 0.0457 & 0.0533 & 0.0903 & 0.0117 & 0.0460 \\
\hline \multirow[t]{2}{*}{$\tau=0.30$} & $Q S T$ & 0.0577 & 0.0520 & 0.0487 & 0.0677 & 0.0717 & 0.0490 & 0.0590 & 0.0547 & 0.0463 & 0.0677 & 0.2123 & 0.0553 & 0.0497 & 0.0497 & 0.0470 & 0.0653 & 0.1183 & 0.0437 \\
\hline & $Q O T$ & 0.0520 & 0.0487 & 0.0473 & 0.0723 & 0.0130 & 0.0467 & 0.0560 & 0.0560 & 0.0467 & 0.0723 & 0.0027 & 0.0593 & 0.0533 & 0.0513 & 0.0517 & 0.1180 & 0.0047 & 0.0477 \\
\hline \multirow{2}{*}{$\tau=0.50$} & $Q S T$ & 0.0493 & 0.0537 & 0.0467 & 0.0503 & 0.0830 & 0.0573 & 0.0527 & 0.0497 & 0.0560 & 0.0503 & 0.2140 & 0.0460 & 0.0463 & 0.0457 & 0.0470 & 0.0663 & 0.1147 & 0.0470 \\
\hline & QOT & 0.0543 & 0.0457 & 0.0507 & 0.0720 & 0.0117 & 0.0547 & 0.0603 & 0.0483 & 0.0443 & 0.0720 & 0.0007 & 0.0570 & 0.0513 & 0.0433 & 0.0480 & 0.1147 & 0.0053 & 0.0493 \\
\hline \multirow[t]{2}{*}{$\tau=0.70$} & $Q S T$ & 0.0557 & 0.0457 & 0.0553 & 0.0580 & 0.0743 & 0.0527 & 0.0507 & 0.0477 & 0.0560 & 0.0580 & 0.2080 & 0.0607 & 0.0493 & 0.0450 & 0.0487 & 0.0637 & 0.1037 & 0.0440 \\
\hline & $Q O T$ & 0.0507 & 0.0450 & 0.0470 & 0.0753 & 0.0123 & 0.0533 & 0.0560 & 0.0500 & 0.0470 & 0.0753 & 0.0023 & 0.0530 & 0.0450 & 0.0410 & 0.0463 & 0.1123 & 0.0043 & 0.0423 \\
\hline \multirow[t]{5}{*}{$\tau=0.90$} & $Q S T$ & 0.0520 & 0.0490 & 0.0583 & 0.0793 & 0.0640 & 0.0570 & 0.0587 & 0.0540 & 0.0543 & 0.0793 & 0.1313 & 0.0487 & 0.0493 & 0.0457 & 0.0547 & 0.0657 & 0.0910 & 0.0480 \\
\hline & $Q O T$ & 0.0463 & 0.0453 & 0.0440 & 0.0550 & 0.0133 & 0.0493 & 0.0503 & 0.0520 & 0.0490 & 0.0550 & 0.0040 & 0.0457 & 0.0550 & 0.0437 & 0.0513 & 0.0897 & 0.0087 & 0.0450 \\
\hline & $F R_{1}$ & 0.0003 & 0.0013 & 0.0007 & 0.0003 & 0.0000 & 0.0000 & 0.0000 & 0.0000 & 0.0000 & 0.0000 & 0.0000 & 0.0013 & 0.0003 & 0.0000 & 0.0010 & 0.0000 & 0.0000 & 0.0023 \\
\hline & $F R_{2}$ & 0.0173 & 0.0187 & 0.0140 & 0.0143 & 0.0003 & 0.0480 & 0.0123 & 0.0120 & 0.0117 & 0.0130 & 0.0000 & 0.0516 & 0.0143 & 0.0180 & 0.0167 & 0.0210 & 0.0000 & 0.0470 \\
\hline & $F R_{3}$ & 0.1230 & 0.0777 & 0.0707 & 0.0097 & 0.0023 & 0.0530 & 0.1090 & 0.0683 & 0.0667 & 0.0003 & 0.0000 & 0.0523 & 0.0897 & 0.0680 & 0.0620 & 0.0000 & 0.0000 & 0.0490 \\
\hline Student's $t$ & & \multicolumn{6}{|c|}{ sample size 200} & \multicolumn{6}{|c|}{ sample size 500} & \multicolumn{6}{|c|}{ sample size 1000} \\
\hline Distribution & & $\mathrm{I}$ & II & III & IV & $\mathrm{V}$ & $\mathrm{VI}$ & $\mathrm{I}$ & II & III & IV & $\mathrm{v}$ & $\mathrm{VI}$ & $\mathrm{I}$ & II & III & IV & $\mathrm{V}$ & VI \\
\hline \multirow[t]{2}{*}{$\tau=0.10$} & $Q S T$ & 0.0970 & 0.0623 & 0.0567 & 0.0737 & 0.1113 & 0.0610 & 0.0607 & 0.0580 & 0.0580 & 0.0883 & 0.1593 & 0.0523 & 0.0640 & 0.0580 & 0.0580 & 0.1413 & 0.2080 & 0.0627 \\
\hline & $Q O T$ & 0.0360 & 0.0470 & 0.0490 & 0.0940 & 0.0243 & 0.0360 & 0.0513 & 0.0623 & 0.0647 & 0.1750 & 0.0107 & 0.0400 & 0.0430 & 0.0623 & 0.0647 & 0.2750 & 0.0060 & 0.0460 \\
\hline \multirow[t]{2}{*}{$\tau=0.30$} & $Q S T$ & 0.0973 & 0.0537 & 0.0650 & 0.0700 & 0.0680 & 0.0587 & 0.0557 & 0.0597 & 0.0567 & 0.1330 & 0.1020 & 0.0587 & 0.0523 & 0.0597 & 0.0567 & 0.2210 & 0.1443 & 0.0673 \\
\hline & $Q O T$ & 0.0360 & 0.0477 & 0.0460 & 0.1203 & 0.0167 & 0.0443 & 0.0400 & 0.0587 & 0.0577 & 0.2570 & 0.0083 & 0.0450 & 0.0303 & 0.0587 & 0.0577 & 0.4320 & 0.0050 & 0.0547 \\
\hline \multirow[t]{2}{*}{$\tau=0.50$} & $Q S T$ & 0.0933 & 0.0443 & 0.0500 & 0.0823 & 0.0663 & 0.0637 & 0.0543 & 0.0520 & 0.0590 & 0.1387 & 0.0803 & 0.0573 & 0.0480 & 0.0520 & 0.0590 & 0.2530 & 0.1207 & 0.0523 \\
\hline & $Q O T$ & 0.0333 & 0.0503 & 0.0503 & 0.1357 & 0.0220 & 0.0463 & 0.0393 & 0.0563 & 0.0540 & 0.2813 & 0.0080 & 0.0433 & 0.0260 & 0.0563 & 0.0540 & 0.4587 & 0.0047 & 0.0523 \\
\hline \multirow[t]{2}{*}{$\tau=0.70$} & $Q S T$ & 0.0923 & 0.0480 & 0.0510 & 0.0867 & 0.0697 & 0.0717 & 0.0527 & 0.0547 & 0.0523 & 0.1320 & 0.0923 & 0.0733 & 0.0573 & 0.0547 & 0.0523 & 0.2443 & 0.1333 & 0.0573 \\
\hline & $Q O T$ & 0.0444 & 0.0483 & 0.0480 & 0.1237 & 0.0230 & 0.0473 & 0.0400 & 0.0570 & 0.0570 & 0.2480 & 0.0113 & 0.0487 & 0.0317 & 0.0570 & 0.0570 & 0.4327 & 0.0053 & 0.0543 \\
\hline \multirow[t]{5}{*}{$\tau=0.90$} & $Q S T$ & 0.102 & 0.0650 & 0.0587 & 0.0927 & 0.0950 & 0.0827 & 0.0610 & 0.0670 & 0.0600 & 0.1030 & 0.1367 & 0.0753 & 0.0537 & 0.0670 & 0.0600 & 0.1640 & 0.2013 & 0.0733 \\
\hline & $Q O T$ & 0.0446 & 0.0513 & 0.0590 & 0.0867 & 0.0217 & 0.0380 & 0.0507 & 0.0600 & 0.0643 & 0.1663 & 0.0167 & 0.0450 & 0.0433 & 0.0600 & 0.0643 & 0.2877 & 0.0080 & 0.0473 \\
\hline & $F R_{1}$ & 0.0513 & 0.0513 & 0.0563 & 0.0673 & 0.0260 & 0.0087 & 0.0660 & 0.0690 & 0.0657 & 0.1047 & 0.0253 & 0.0090 & 0.0650 & 0.0737 & 0.0890 & 0.1393 & 0.0200 & 0.0067 \\
\hline & $F R_{2}$ & 0.1110 & 0.1163 & 0.1213 & 0.1670 & 0.0570 & 0.0517 & 0.1410 & 0.1613 & 0.1523 & 0.2553 & 0.0473 & 0.0567 & 0.1387 & 0.1563 & 0.1707 & 0.3497 & 0.0330 & 0.0527 \\
\hline & $F R_{3}$ & 0.2137 & 0.1767 & 0.1770 & 0.0390 & 0.0803 & 0.0640 & 0.2447 & 0.2140 & 0.2053 & 0.0143 & 0.0560 & 0.0607 & 0.2580 & 0.1973 & 0.2123 & 0.0057 & 0.0357 & 0.0590 \\
\hline Skew $t$ & & \multicolumn{6}{|c|}{ sample size 200} & \multicolumn{6}{|c|}{ sample size 500} & & & sample & ze 1000 & & \\
\hline Distribution & & $\mathrm{I}$ & II & III & IV & $\mathrm{V}$ & VI & I & II & III & IV & $\mathrm{V}$ & $\mathrm{VI}$ & $\mathrm{I}$ & II & III & IV & $\mathrm{V}$ & $\mathrm{VI}$ \\
\hline$\tau=0.10$ & QST & 0.0487 & 0.0470 & 0.0550 & 0.0703 & 0.0467 & 0.0483 & 0.0517 & 0.0407 & 0.0533 & 0.0600 & 0.0407 & 0.0540 & 0.0540 & 0.0507 & 0.0480 & 0.0647 & 0.0460 & 0.0490 \\
\hline & QOT & 0.0357 & 0.0343 & 0.0420 & 0.0443 & 0.0357 & 0.0313 & 0.0413 & 0.0400 & 0.0413 & 0.0650 & 0.0390 & 0.0397 & 0.0453 & 0.0427 & 0.0453 & 0.0890 & 0.0423 & 0.0413 \\
\hline$\tau=0.30$ & QST & 0.0473 & 0.0540 & 0.0487 & 0.0633 & 0.0483 & 0.0617 & 0.0527 & 0.0450 & 0.0530 & 0.0693 & 0.0433 & 0.0527 & 0.0513 & 0.0527 & 0.0493 & 0.0847 & 0.0490 & 0.0440 \\
\hline & QOT & 0.0493 & 0.0373 & 0.0420 & 0.0830 & 0.0443 & 0.050 & 0.0483 & 0.0497 & 0.0437 & 0.1127 & 0.0380 & 0.0513 & 0.0493 & 0.0487 & 0.0500 & 0.1683 & 0.0380 & 0.0510 \\
\hline$\tau=0.50$ & QST & 0.0490 & 0.0557 & 0.0467 & 0.0637 & 0.0567 & 0.0613 & 0.0513 & 0.0477 & 0.0440 & 0.0990 & 0.0510 & 0.0470 & 0.0533 & 0.0477 & 0.0527 & 0.1187 & 0.0577 & 0.0553 \\
\hline & QOT & 0.0413 & 0.0477 & 0.0507 & 0.0920 & 0.0463 & 0.0543 & 0.0427 & 0.0433 & 0.0457 & 0.1640 & 0.0403 & 0.0480 & 0.0463 & 0.0483 & 0.0523 & 0.2383 & 0.0377 & 0.0527 \\
\hline$\tau=0.70$ & QST & 0.0563 & 0.0627 & 0.0530 & 0.0653 & 0.0507 & 0.0680 & 0.0530 & 0.0553 & 0.0540 & 0.1010 & 0.0560 & 0.0567 & 0.0603 & 0.0507 & 0.0540 & 0.1593 & 0.0557 & 0.0523 \\
\hline & QOT & 0.0477 & 0.0523 & 0.0553 & 0.0993 & 0.0440 & 0.0573 & 0.0440 & 0.0560 & 0.0500 & 0.1953 & 0.0303 & 0.0527 & 0.0497 & 0.0500 & 0.0543 & 0.2987 & 0.0340 & 0.0493 \\
\hline$\tau=0.90$ & QST & 0.0777 & 0.0617 & 0.0597 & 0.0963 & 0.0577 & 0.070 & 0.0557 & 0.0597 & 0.0607 & 0.0987 & 0.0540 & 0.0587 & 0.0577 & 0.0523 & 0.0610 & 0.1587 & 0.0663 & 0.0517 \\
\hline & $\mathrm{QOT}$ & 0.0480 & 0.0500 & 0.0540 & 0.0867 & 0.0350 & 0.0543 & 0.0447 & 0.0527 & 0.0533 & 0.1710 & 0.0290 & 0.0447 & 0.0520 & 0.0597 & 0.0660 & 0.2810 & 0.0187 & 0.0467 \\
\hline
\end{tabular}


Table 4: Descriptive Statistics of Countries in the Asia Crisis

\begin{tabular}{|c|c|c|c|c|c|c|c|c|}
\hline & Mean & Variance & Median & Skewness & $\begin{array}{c}\text { Excess } \\
\text { Kurtosis }\end{array}$ & $\begin{array}{c}J-B^{1} \\
\text { Test }\end{array}$ & $\begin{array}{c}\text { Ljung }- \text { Box }{ }^{1} \\
\text { Box Test } \\
\end{array}$ & $\begin{array}{c}A R C H^{1} \\
\text { Test }\end{array}$ \\
\hline Hong Kong & -0.00142 & 0.000673 & 0 & 0.379216 & 6.056046 & $1511.839991^{* 2}$ & $26.73796242^{*}$ & $102.3380846^{*}$ \\
\hline South Korea & -0.00171 & 0.000784 & 0 & 0.137872 & -0.97908 & $76.94547603^{*}$ & $17.35117772^{*}$ & $56.36415268^{*}$ \\
\hline Malaysia & -0.00324 & 0.000672 & -0.00269 & 1.541682 & 9.571008 & $3063.658555^{*}$ & 9.091541876 & 3.9842208 \\
\hline Japan & -0.00036 & 0.000138 & 0 & 0.021421 & -0.08357 & $156.7419826^{*}$ & $11.8900159^{*}$ & $46.32324159^{*}$ \\
\hline U.K. & 0.000559 & 0.0000997 & 0.000517 & -0.2638 & -2.14174 & $18.94853472^{*}$ & $20.90735135^{*}$ & $33.27060309^{*}$ \\
\hline U.S. & 0.00036 & 0.00013 & 0.000546 & -1.13983 & 3.963259 & $983.0770432^{*}$ & 1.818650886 & $29.16018572^{*}$ \\
\hline Australia & 0.0000512 & 0.000546 & 0.00092 & -0.57487 & 2.63192 & $605.8060695^{*}$ & 3.054880627 & $117.825644^{*}$ \\
\hline Germany & 0.001126 & 0.000215 & 0.00225 & -0.86824 & 0.614144 & $295.1270798^{*}$ & $10.40811878^{*}$ & $67.6223594^{*}$ \\
\hline Thailand & -0.00312 & 0.000588 & -0.00388 & 0.94095 & 0.220109 & $255.3603359^{*}$ & $19.25763484^{*}$ & $65.01672425^{*}$ \\
\hline
\end{tabular}

Notes:

${ }^{1}$ In the above table, the values of the Jarque-Bera test, the Ljung-Box test and the ARCH LM test are calculated with $\operatorname{lag} s=5$.

$2 *$ indicates the rejection of the null hypothesis. 
Table 5: Correlations Among Several Countries in the Asian Crisis (Before Crisis ${ }^{1}$ )

\begin{tabular}{|c|c|c|c|c|c|c|c|c|c|}
\hline & Hong Kong & South Korea & Malaysia & Japan & U.K. & U.S.j & Australia & Germany & Thailand \\
\hline Hong Kong & 1 & & & & & & & & \\
\hline South Korea & 0.151735 & 1 & & & & & & & \\
\hline Malaysia & 0.273213 & 0.047137 & 1 & & & & & & \\
\hline Japan & 0.308531 & 0.031992 & 0.088048 & 1 & & & & & \\
\hline U.K. & 0.151702 & 0.065907 & -0.01891 & 0.130064 & 1 & & & & \\
\hline U.S. & 0.081478 & 0.008263 & 0.001455 & -0.04569 & 0.331209 & 1 & & & \\
\hline Australia & 0.273855 & 0.05636 & 0.137215 & 0.235572 & 0.117007 & -0.01811 & 1 & & \\
\hline Germany & 0.266234 & 0.024454 & 0.101507 & 0.125796 & 0.457624 & 0.242969 & 0.397739 & 1 & \\
\hline Thailand & 0.082663 & 0.036424 & 0.132881 & 0.016986 & 0.023094 & -0.03897 & 0.103829 & 0.04785 & 1 \\
\hline \multicolumn{10}{|c|}{ Table 6: Correlations Among Several Countries in the Asian Crisis (During Crisis²) } \\
\hline & Hong Kong & South Korea & Malaysia & Japan & U.K. & U.S. & Australia & Germany & Thailand \\
\hline Hong Kong & 1 & & & & & & & & \\
\hline South Korea & 0.130589 & 1 & & & & & & & \\
\hline Malaysia & 0.444207 & 0.285872 & 1 & & & & & & \\
\hline Japan & 0.399132 & 0.13909 & 0.296769 & 1 & & & & & \\
\hline U.K. & 0.489817 & 0.204314 & 0.290062 & 0.396905 & 1 & & & & \\
\hline U.S. & 0.261643 & 0.169513 & 0.10811 & 0.136181 & 0.518994 & 1 & & & \\
\hline Australia & 0.589521 & 0.261065 & 0.36159 & 0.490537 & 0.449319 & 0.190915 & 1 & & \\
\hline Germany & 0.622381 & 0.172951 & 0.291964 & 0.394909 & 0.684973 & 0.396108 & 0.568798 & 1 & \\
\hline Thailand & 0.442736 & 0.328666 & 0.479853 & 0.241142 & 0.278739 & 0.137684 & 0.380339 & 0.268605 & 1 \\
\hline
\end{tabular}

${ }^{1}$ Before crisis: from January 1, 1997 to October 19, 1997. [2] After crisis: from October 20, 1997 to August 31,1998 